\title{
Influence of Different Populations on Pharmacokinetic Bioequivalence Results: Can We Extrapolate Bioequivalence Results from One Population to Another?
}

Deniz Ozdin ${ }^{1,2}$, France Varin ${ }^{1}$, Anders Fuglsang ${ }^{3}$, Dina Al-Numani², Murray P. Ducharme ${ }^{1,2}$

${ }^{1}$ Faculté de Pharmacie, University of Montreal, Montreal, QC, Canada; ${ }^{2}$ Learn and Confirm Inc., St-Laurent, QC, Canada; ${ }^{3}$ Fuglsang Pharma, Haderslev, Denmark

\begin{abstract}
Purpose: Over the last 15 years, an ever-increasing proportion of pharmacokinetic bioequivalence studies for European/North American generic submissions appeared to have been conducted in geographical/ethnic populations other than those for which the drug is marketed for. The results of pharmacokinetic bioequivalence studies have traditionally been considered to be insensitive to the population studied. However, several recent studies have suggested that this may not necessarily be true. The objective of this study was to investigate whether there were any concerns regarding the current practice of extrapolating bioequivalence study results from one geographic/ethnic population to another. Methods: In order for a regulatory agency to use bioequivalence results from one population to another, two formulations assessed as bioequivalent under fasted and fed conditions in one population must be bioequivalent in a geographically/ethnically different population under both conditions. Unfortunately, bioequivalence studies between a generic and its reference product for one submission are conducted using only one geographical/ethnic population. As bioequivalence study results between two populations for the same generic and reference products are not available, the food effect for the same reference product between two populations was compared. This is based on the rationale that if two products are bioequivalent under both fasted and fed conditions in two populations, even if there are PK differences in the product exposures between these two populations, the test to reference ratio, as well as the food effect, will remain constant within each population. Food effect (fed/fasted ratio) was calculated using pharmacokinetic data from publicly available regulatory resources and compared between two geographical/ethnic populations using the same reference for each studied drug product. Meta-analyses were conducted. Results: Statistically significant differences $(P<0.05)$ were found in the food effect between two populations for nine out of the ten $(90 \%)$ available studied products. Among these, an observed clinical difference was suggested in three out of nine $(33 \%)$ products. Conclusion: These results suggest that bioequivalence results from one population may not always be representative of what may be found in another population.
\end{abstract}

Corresponding author: Murray P. Ducharme, PharmD, FCCP, FCP, Learn and Confirm Inc., 750 Marcel-Laurin, Suite 235, St-Laurent, QC, Canada, H4M 2M4; Tel/Fax, (514) 373-5346; Email,

Murray.Ducharme@learnandconfirm.ca

Received, June 4, 2020; Revised, September 1, 2020; Accepted, July 28, 2020; Published, September 28, 2020

\section{INTRODUCTION}

The bioavailability of orally administered drug products can be altered under different (patho)physiological conditions. To date, different studies in animal models and human subjects have revealed that altered pathophysiological conditions can affect not only the bioavailability of drug products (1-4), but also pharmacokinetic (PK) bioequivalence study outcomes. Drug products that were found to be bioequivalent in one condition were not always bioequivalent in other conditions (5-8). The US Food and Drug Administration (FDA) and other regulatory agencies often require the demonstration of bioequivalence under both fasted and fed conditions for generic submissions (9-11), as formulations may perform differently under different conditions; therefore, two formulations assessed to be bioequivalent under fasted conditions may not necessarily be bioequivalent under fed conditions. Regulatory agencies typically recommend a high-fat, highcaloric meal in food effect (FE) bioavailability 
studies for $\mathrm{NDAs}^{1}$ and fed bioequivalence studies for $\operatorname{ANDAs}^{2}$ (9-11), as bioequivalence would then be investigated in potentially extreme conditions (i.e., fasting and high-fat conditions). A high-fat, high-caloric meal would likely provide maximal gastrointestinal perturbation, rendering the physiological conditions more discriminative at detecting formulation differences.

The population for which the drug is intended to be marketed for has to be part of the population(s) investigated in pivotal Phase III studies. Hence, a new drug cannot be marketed in one population without having pivotal data obtained from the same population. In contrast, generic drugs are currently marketed in different populations from which they were tested in, as a result of cost-reducing measures that enable companies to offer their generic products at a lower cost. It is therefore not surprising to learn that over the last 15 years, most generic products have been for a while tested in India, while they are destined to be marketed in "higher-cost" regions such as Canada, the USA, and Europe. Extrapolation of bioequivalence results from one population to another has been considered to be a non-issue for many decades now, and is actually the reason for which bioequivalence studies are typically conducted in healthy volunteers (HVs) instead of patients in the first place. This is based on the premise that (a) PK bioequivalence studies are usually conducted in a crossover fashion and, therefore, subjects will act as their own control, and so even if they would present different PK characteristics, these differences would apply equally to both crossover periods and therefore products, and that (b) bioequivalence involves the assessment of the relative bioavailability of a test formulation in one period versus a reference one in another period of the study, and is expressed as a ratio.

We are noting, however, that the above would only hold true if the following two conditions are met: (a) absorption would be a unidirectional passage of drug molecules from intestinal lumen into the portal vein, meaning that once absorbed, an active ingredient or moiety cannot come back into the gut lumen, and (b) excipients in test and reference formulations are exactly the same qualitatively and quantitatively (Q1/Q2) or, if they are different, they cannot produce a different effect on metabolizing enzymes and transporters in the gut wall between the two formulations.

Regarding the unidirectional passage of drug molecules, influx and efflux transporters localized in the enterocyte membranes have been shown to be key determinants of drug absorption, regulating the transport of drug molecules from extracellular to the intracellular environment, and vice versa (12-14). Once a drug is absorbed from the intestinal lumen into the enterocytes, efflux transporters at the apical membrane of enterocytes may drive it from inside the cell back into the lumen, thus "reversing" its absorption through the gut wall and its subsequent entry into the portal vein. This process can be repeated multiple times, predominantly in the small intestine, and eventually determines the fraction of drug that will be absorbed. Therefore, contrary to the main general assumption, absorption is not always unidirectional and an active ingredient or moiety that is absorbed can be "de-absorbed" and come back into the gut lumen and may therefore interact further with components there such as food and excipients.

As for the excipients, an oral generic formulation does not generally have to contain the same inactive ingredients as the reference product (15). Excipients are traditionally used in part to facilitate drug release and dissolution which are essential precursor steps for drug absorption. Their impact on bioavailability, and in turn bioequivalence outcomes, has been traditionally assumed to be negligible. However, accumulating evidence is showing that many excipients can impact bioavailability and bioequivalence outcomes, not only by modulating drug release and dissolution, but also by their inhibitory/inductive effect on CYP enzymes and transporters that are present in the gut wall and elsewhere (16-18). Indeed, many studies have revealed that the presence of some excipients in one formulation, but not in another, had unexpected impact on the bioavailability of the drug product, and hence caused nonbioequivalence (19-21).

\section{${ }^{2}$ Abbreviated New Drug Application}


In summary, neither of the two necessary conditions are met to support the traditional view that the studied population should not impact bioequivalence results. Therefore, bioequivalence outcomes between two populations need to be further studied.

The necessity of further investigation is also amplified by evidence in the literature suggesting that different populations may present varying levels of expression and prevalence of functional variants of transporters and CYP enzymes. For example, certain transporters and CYP enzymes have been reported to be markedly lower in Asian/Indian and African American populations compared to a North American/ Caucasian one (22-26). If that is truly the case, then transporters/enzymes may theoretically play a less important role in drug disposition in an Indian population, and the impact of any interaction between enzymes/transporters, food and/or excipients on drug bioavailability may be expected to be smaller. Should gut transporters and/or enzymes be involved in FE of some drugs, one could then hypothesize that a different $\mathrm{FE}$ would be expected in an Indian population versus a North American one for these drug products. Indeed, the effect of food has been shown to be influenced by transporters/CYP enzymes, as many studies have demonstrated that the PK differences seen for some drug products with food are due to interactions between food constituents and transporters/ CYP enzymes (27-30).

Using the above discussed concepts that (1) drugs can be absorbed and then "de-absorbed" by transporters, (2) different populations may have different transporter and enzyme expression in the gut wall, and that (3) the presence of food constituents and excipients can affect transporter and enzyme expression, one can hypothesize that drug bioavailability or PK equivalence between test and reference in different populations may be influenced by the interaction between food constituents, drug excipients, and transporters/ enzymes. This hypothesis would be in contradiction with the historical common viewpoint that populations do not matter in the assessment of bioequivalence. It would then maybe possible that changes in physiological factors between populations would result in different test/reference ratios between them. As a consequence, bioequivalence study results would potentially be different between two different populations.

This is exemplified in the hypothetical situation depicted in Figure 1, where bioequivalence between a test and a reference product is investigated in two different populations. These two populations differ in their level of expression or activity of transporters/enzymes for the hypothetical drug product. For simplicity purposes, let us consider that Population 1 (Pop 1) has no enzymes/transporters expression in the gut wall, while Population 2 (Pop 2) has a high level. In addition, let us also assume that the FE involves only interactions between food constituents and transporters. In this hypothetical situation, an FE will not be present in Pop 1, but will be present in Pop 2. Should the test and reference be bioequivalent under fasted conditions in Pop 1, they will automatically also be bioequivalent under fed conditions. For Pop 2, even if the test and reference are bioequivalent under fasted conditions, they may not be bioequivalent at all under fed. In summary, Figure 1 describes how a different FE will be observed between two populations leading to different BE outcomes.

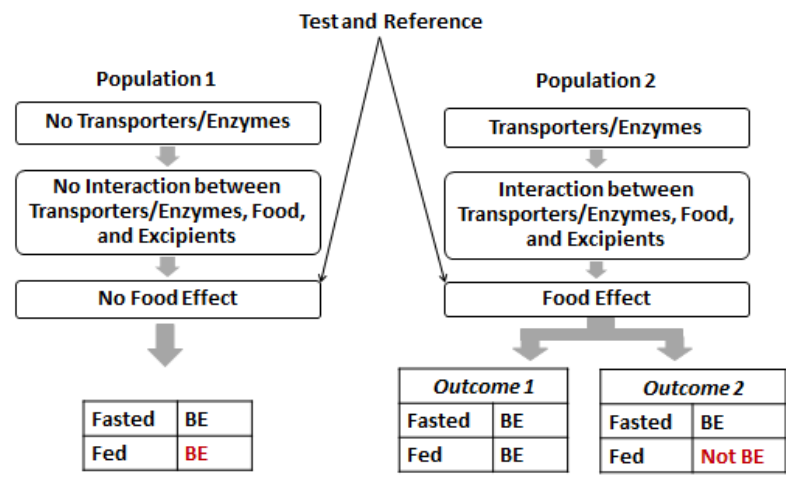

Figure 1. The hypothetical outcomes of conducting pharmacokinetic bioequivalence studies in populations with different levels of expression or activity of CYP enzymes and/ or transporters. Here the two formulations are BE in Population 1 (with no food effect) under both fasted and fed conditions, but may not be BE in Population 2 (with food effect) under fed conditions (Outcome 2). 
To allow the extrapolation of bioequivalence outcomes from one population to the next, the test and reference products do not need to have the same bioavailability between different populations, but the FE of each formulation will need to be identical between two populations. This can be better comprehended by an example which is depicted in Figure 2. The same test and reference products are given to two different populations, Pop 1 and 2, under both fasted and fed conditions, with hypothetical bioavailability values. Due to the influence of altered physiological conditions, the bioavailability (expressed as area under the curve, AUC) of the products is different between the two populations, and also under the fed and fasted conditions. For Pop 1, AUC of both the test and reference is $100 \mathrm{ng} \mathrm{h} \mathrm{mL}^{-1}$ under fasted versus $200 \mathrm{ng} \mathrm{h} \mathrm{mL} \mathrm{mL}^{-1}$ under fed conditions. For Pop 2, AUC is $50 \mathrm{ng} \mathrm{h} \mathrm{mL}^{-1}$ under fasted versus $100 \mathrm{ng} \mathrm{h} \mathrm{mL} \mathrm{m}^{-1}$ under fed conditions. As shown in Figure 2, while the AUC is different between the populations, the ratio of fed/fasted for both populations remains the same i.e., 2, implying the same FE is present for both populations. This example explains further that if test and reference products are bioequivalent under both fasted and fed conditions in two different populations, the FE for these products has to be also the same between these two populations.

The main objective of this project is, therefore, to investigate whether bioequivalence study results from one ethnic population can be extrapolated to another one (different in ethnicity, but both consisting of HVs). In order to extrapolate bioequivalence results from one population to another, two formulations assessed as bioequivalent in one population must be bioequivalent in another one as well. In this retrospective study, it was impossible to compare bioequivalence study results of the same generic versus reference products in two different populations as generic firms only conduct their pivotal bioequivalence studies once in one population. Using the above discussed concept that for two products to be bioequivalent in two different populations the FE also has to be the same, we compared the FE results in lieu of bioequivalence results for a given reference product between different populations. Food effect diffedtentin populations can be derived as some sponsors conduct their bioequivalence studies in one population (e.g., in North America) while others conduct them in another one (e.g., India) for the same reference product. The FE results are also a better measure as PK results may vary from one population to the next, so the mean exposure results, i.e., AUC and maximum observed concentration $\left(\mathrm{C}_{\max }\right)$, cannot be compared across populations. Comparing FE results, which are the exposure ratios of fed to fasted conditions, for the same reference product, allows for appropriate comparisons between two populations. In this study, FE results for several reference drug products between two or more different geographical/ethnic $\mathrm{HV}$

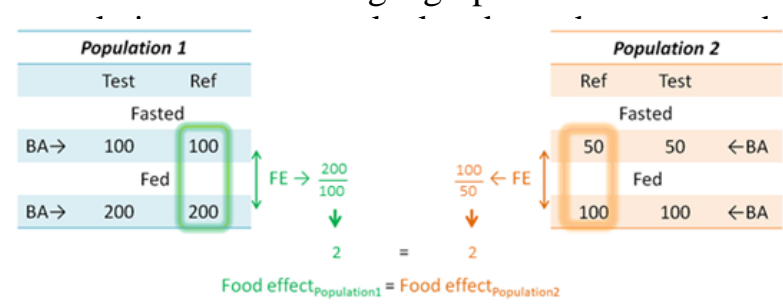

Figure 2. An example illustrating the same food effect between two ethnic/geographical populations despite different bioavailabilities of drug products under altered physiological conditions (fed vs. fasted; Population 1 vs. Population 2) when they are bioequivalent in both populations. BA, Bioavailability; Ref, Reference.

\section{METHODS}

\section{Data Extraction}

A literature search for drug products with fasted and fed bioequivalence data was conducted using Health Canada's Drug Product Database Online Query (31). All available product monographs by different manufacturers for oral pharmaceutical dosage forms (i.e., immediate-release and modified-release tablets and capsules) of each active pharmaceutical ingredient (API) were screened. Only those providing the complete list of required information were included for FE calculations. The required information was extracted from Part II (Scientific Information), Clinical Trials subsection of product 
monographs, Comparative Bioavailability Studies (Fasted and Fed) in Abbreviated New Drug Submissions (ANDSs), and only for reference product (US Reference Listed Drug or Canadian Reference Product). In parallel, the US FDA online database for FDA Approved Drug Products, Drugs@FDA (32); FDA's Clinical Pharmacology, Biopharmaceutics and Statistical Reviews in NDA of each drug were reviewed for FE bioavailability study results. When available, fed and fasted data were also extracted from FE bioavailability studies in NDAs.

Required information consisted of the following:

1. Population geometric means of $\ln$ transformed $\mathrm{AUC}_{0-\mathrm{t}}$ and $\mathrm{C}_{\max }$

2. Inter-individual variability (inter-CV\%) of each PK parameter from both fasted and fed comparative bioavailability studies

3. Studied population

4. Subject numbers

5. Date and site of the study

The labels of US Reference Listed Drug (RLD) and monographs of Canadian Reference Products were compared to ensure that they were exactly the same products when data from both US and Canadian reference drug products were used.

The data was publicly available for two populations. Indian population was selected as Population 1 for which the studies were conducted in India, and North American population was selected as Population 2 for which the studies were conducted in Canada or in the US.

\section{Assessment of Food Effect for Each ANDA/ANDS Study}

Effect of food (FE) on bioavailability was assessed by calculating fed/fasted ratios of population geometric means (GMR) of $1 n$ transformed $\mathrm{PK}$ parameters $\left(\mathrm{AUC}_{0-\mathrm{t}}\right.$ and $\mathrm{C}_{\max }$ ) and the $90 \%$ confidence intervals (CIs) for these ratios using $\mathrm{AUC}_{0-\mathrm{t}}$ and $\mathrm{C}_{\max }$ of reference from Comparative Bioavailability Studies (Fasted and $\mathrm{Fed}$ ) in the same ANDS. Since the fasted and fed PK data were collected from two independent groups of subjects with unbalanced sample sizes, the CI equation was adapted to an unbalanced parallel design via a pooled standard deviation estimate (33):

$$
\begin{array}{r}
\mathrm{Cl}=\operatorname{Exp}\left[\left(\operatorname{Ln} \frac{\mu_{f e d}}{\mu_{f a s t e d}}\right) \pm\right. \\
\left.. S D_{\text {pooled }} \sqrt{\frac{n_{\text {fed }}+n_{\text {fasted }}}{n_{\text {fed }} \cdot n_{\text {fasted }}}}\right]
\end{array}
$$

where $\mu_{f e d}$ and $\mu_{\text {fasted }}$ are population geometric means of the PK parameter under fed and fasted, respectively, $\frac{\mu_{\text {fed }}}{\mu_{\text {fasted }}}$ represents the point estimate $(\mathrm{PE})$, and $t_{1-\alpha, n_{\text {fed }}+n_{\text {fasted }}-2}$ denotes the critical value of the $t$ distribution with $n_{\text {fed }}+n_{\text {fasted }}-$ 2 degrees of freedom at the $1-\alpha$ probability level. The $\alpha=0.05$ was chosen based on the Two One-Sided Tests (TOST) procedure (34). $S D_{\text {pooled }}$ is the pooled standard deviation which is calculated as the square root of the pooled error variance $\left(\sigma^{2}\right.$ pooled $)$ estimate:

$$
\begin{aligned}
S D_{\text {pooled }}=\sqrt{\sigma_{\text {pooled }}^{2}}= \\
\sqrt{\frac{\left[\left(n_{f e d}-1\right) \sigma^{2} f e d\right]+\left[\left(n_{f a s t e d}-1\right) \sigma^{2} \text { fasted }\right]}{\left(n_{f e d}+n_{f a s t e d}-2\right)}}
\end{aligned}
$$

where $\sigma^{2}$ fed and $\sigma_{\text {fasted }}^{2}$ are the variance estimates associated with the fed and fasted treatments, respectively. The variance associated with each treatment was calculated from the interindividual variability (CV\%) provided for reference in that treatment. To convert between a variance $\left(\sigma^{2}\right)$ on the log scale and a $\mathrm{CV}$ on the observed scale, the following relation was applied:

$$
\sigma^{2}=\operatorname{Ln}\left(C V^{2}+1\right)
$$

\section{Assessment of Summary Food Effect in Each Population}

Fixed-effect and random-effects model metaanalyses were performed to assess the summary $\mathrm{FE}$ for each drug in each region/population in terms of GMRs (fed/fasted) for $\mathrm{C}_{\max }$ and $\mathrm{AUC}_{0-\mathrm{t}}$ and the $95 \% \mathrm{CI}$ for the ratios, using the FEs calculated from all ANDS studies available in that population. Meta-analytic computations were performed manually (35) using Microsoft Excel $2010^{\circledR}$. 
Provided that the PK of the drug substance was linear, studies with different dose strengths for the same reference product were included in the meta-analysis for the calculation of the summary FE. When more than one reference was available for a drug, a literature search was performed to investigate the bioequivalence between the reference products. Only the same (bioequivalent) reference products were included in the same meta-analysis for the calculation of the summary FE.

\section{Comparison of Food Effect between North American and Indian Populations for Significance}

The summary FEs for the same reference products were compared between the North American and Indian populations for a significant difference from both statistical and clinical standpoints.

Statistical significance: A subgroup analysis was performed to compare the summary FEs between Indian and North American subgroups for statistical significance using a Q-test based on analysis of variance. The difference in summary FE between the two populations was concluded to be statistically significant when $P<0.05$ for either of the PK parameters.

Clinical significance: A difference of $\geq 40 \%$ between the FEs of the North American and

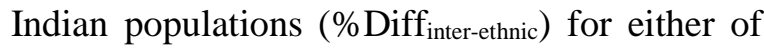
the PK parameters was considered to be of possible clinical relevance. When food impacted exposure in the same direction in both populations, the difference was calculated as: $\%$ Diff $_{\text {inter-ethnic }}=\quad \mid \% \mathrm{FE}_{\text {North American }}-$ $\% \mathrm{FE}_{\text {Indian }} \mid$. When food impacted exposure in two different directions, the difference was calculated as:

$\%$ Diff $_{\text {inter-ethnic }}=\left|\% \mathrm{FE}_{\text {North American }}\right|+\left|\% \mathrm{FE}_{\text {Indian }}\right|$.

\section{Between-Study Variability in Food Effect}

Between-study variability in FEs of different studies in each population was assessed for each study drug using heterogeneity measures, tausquared $\left(\tau^{2}\right)$ and $I^{2}$ indices. $I^{2}$ of $25 \%, 50 \%$, and $75 \%$ were considered as low, moderate, and high heterogeneity, respectively (35). The heterogeneity measure $\left(I^{2}\right)$ was then compared between the two populations.

\section{RESULTS}

Figure 3 summarizes the study steps for meeting the objective (FE calculations in two populations and their comparison), as well as for evaluating and comparing heterogeneity in each population. A total of 27 drug candidates with available fasted and fed bioequivalence studies in the literature were selected based on pre-established search criteria; however, only nine active drug ingredients (10 drug products) had all the required information for evaluating $\mathrm{FE}$ in two different geographical/ethnic populations and were included in the data analysis consisting of a total of 53 ANDSs and six NDAs. The list of the nine active drug ingredients, their respective reference products, and their product label's reported $\mathrm{FE}$ are provided in Table 1. The table also includes which CYP enzymes and/or transporters are known at this time to be involved in the metabolism and disposition of these particular drugs (36-39).

Detailed FE results (point estimates and $90 \%$ confidence intervals) are summarized by drug, ANDS, and region in Table 2 for $\mathrm{AUC}_{0-\mathrm{t}}$ and Table 3 for $\mathrm{C}_{\max }$, and only for reference products which had the required information available for summary FE calculations in both populations, enabling the comparison of FEs between them. All other reference products with summary FE in one population, but not the other, were only used in the heterogeneity analysis. In the North American population, $11.1 \%$ and $44.4 \%$ of the studies were associated with heterogeneity above the level of significance $\left(I^{2} \geq 75 \%\right)$ in terms of $\mathrm{AUC}_{0-\mathrm{t}}$ and $\mathrm{C}_{\max }$, respectively, while $71.4 \%$ of the studies in Indian populations had significant between-study variability in terms of both exposure measures (Table 4).

When the FEs for different reference products of a drug were similar (e.g., Nexium ${ }^{\circledR} \quad 20$ and $40 \quad$ mg tablets of esomeprazole; Prilosec $\AA \quad 20 \quad \mathrm{mg}$ capsule, Losec ${ }^{\circledR} 20 \mathrm{mg}$ tablet and capsule of omeprazole), the summary FE was calculated one more time by including all reference products for that drug (Tables 5 and 6). 
Table 7 specifies which reference products were used for the comparison of FEs between the two populations and summarizes the overall FEs (i.e., the ones calculated in this study and those from the reference product labels) per drug and population, and the comparison between the two populations (statistical and clinical relevance).
For nine out of the ten drug products, the FEs were significantly different between North American and Indian populations statistically (Qtest; $P<0.05)$ and for three of these nine the difference could be of possible clinical relevance (\% Diff inter-ethnic $_{\text {in }} \mathrm{FE} \geq 40 \%$ ).

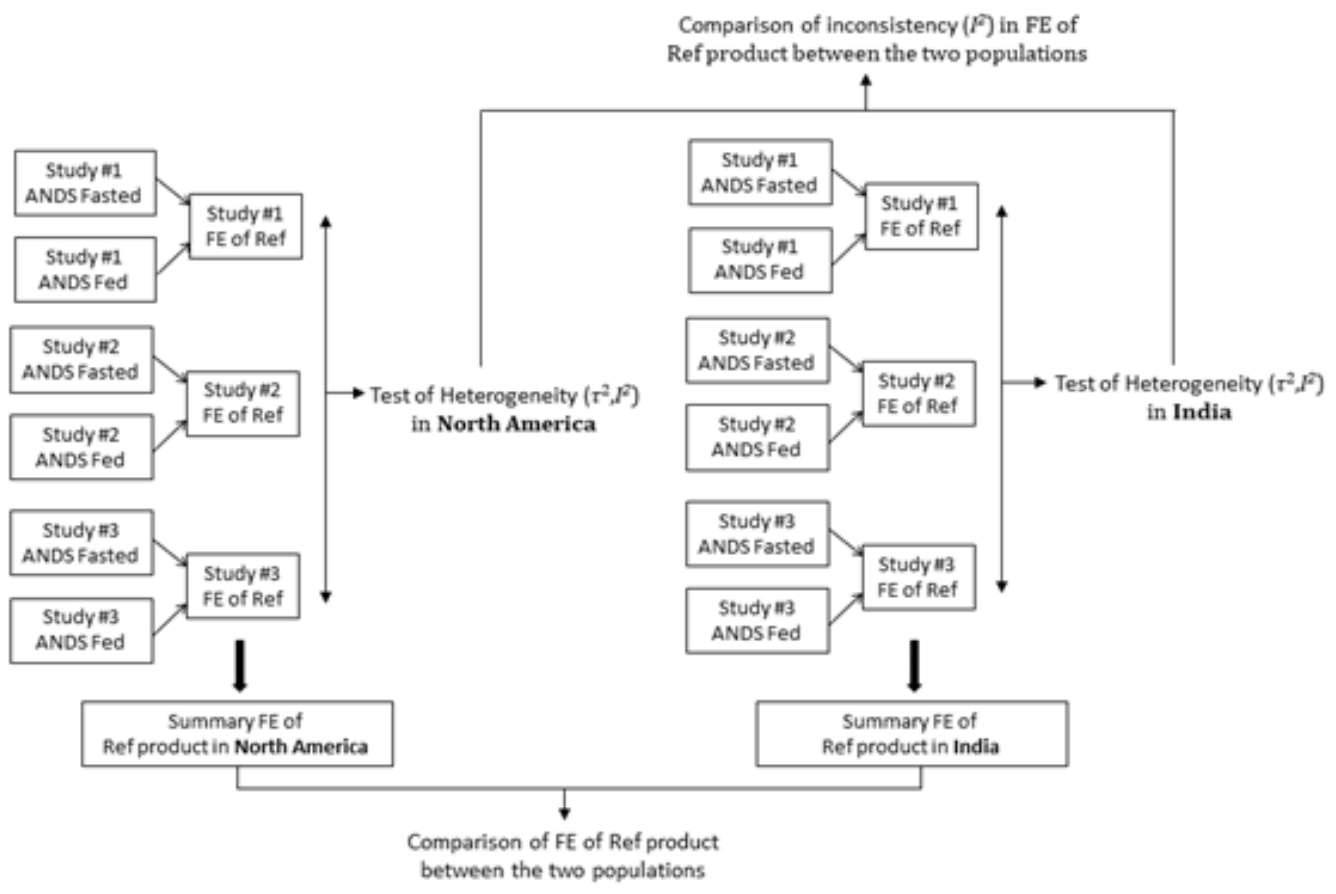

Figure 3 Study flow diagram. ANDS, Abbreviated New Drug Submission; FE, food effect; $\tau^{2}$, measure of heterogeneity (Between-studies variance); $I^{2}$, measure of heterogeneity (degree of inconsistency in \%); Ref, reference. 
Table 1. Included drugs overview

\begin{tabular}{|c|c|c|c|}
\hline$\overline{\text { API }}$ & Reference Product & CYP Enzyme/Transporter $\psi$ & $\begin{array}{l}\text { Designated FE on } \\
\text { the label (range) }\end{array}$ \\
\hline Amiodarone & $\begin{array}{l}\text { Cordarone }{ }^{\circledR} 200 \mathrm{mg} \\
\text { Tab }^{\mathrm{ab}}\end{array}$ & $\begin{array}{l}\text { Major substrate of CYP3A, } \\
\text { CYP2C8 } \\
\text { Minor substrate of CYP2D6, } \\
\text { CYP1A2 } \\
\text { P-gp/ABCB1 }\end{array}$ & $\begin{array}{l}\text { AUC increase x } 2.4 \\
(1.7-3.6) \\
C_{\max } \text { increase x } 3.8 \\
(2.7-4.4)\end{array}$ \\
\hline Carbamazepine & $\begin{array}{l}\text { Tegretol® CR } 400 \mathrm{mg} \\
\text { Tab }^{\mathrm{b}}\end{array}$ & $\begin{array}{l}\text { Major substrate of CYP3A4 } \\
\text { Minor substrate of CYP2C8 }\end{array}$ & No significant FE \\
\hline Diltiazem & 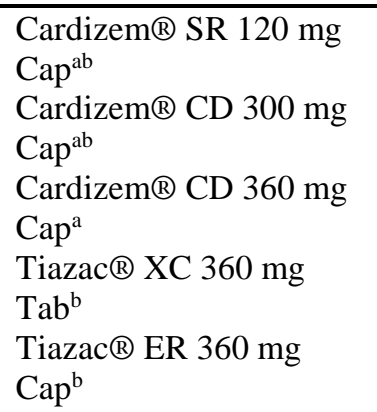 & $\begin{array}{l}\text { Major substrate of CYP3A4 } \\
\text { Minor substrate of CYP2C9, } \\
\text { CYP2D6 } \\
\text { P-gp/ABCB }\end{array}$ & 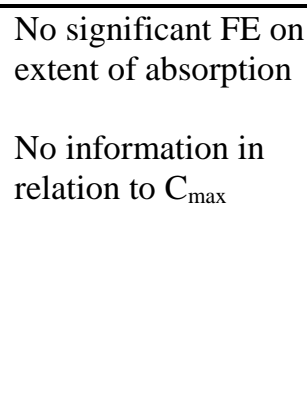 \\
\hline Verapamil & $\begin{array}{l}\text { Isoptin® SR } 240 \mathrm{mg} \\
\text { Tab }^{\text {ab }}\end{array}$ & $\begin{array}{l}\text { Major substrate of CYP3A4 } \\
\text { Minor substrate of CYP1A2, } \\
\text { CYP2C8, CYP2C9, CYP2C18, } \\
\text { CYP2B6, CYP2E1 }\end{array}$ & $\begin{array}{l}\text { AUC decrease by } 1- \\
8 \% \\
\mathrm{C}_{\max } \text { decrease by } \\
15 \% \$\end{array}$ \\
\hline Esomeprazole & $\begin{array}{l}\text { NEXIUM® } 40 \mathrm{mg} \\
\mathrm{Tab}^{\mathrm{ab}} \\
\mathrm{NEXIUM}{ }^{\mathrm{a}} 20 \mathrm{mg} \\
\mathrm{Tab}^{\mathrm{ab}}\end{array}$ & $\begin{array}{l}\text { Major substrate of CYP2C19 } \\
\text { Minor substrate of CYP3A4 }\end{array}$ & $\begin{array}{l}\text { AUC decrease by } \\
43.7 \% \dagger \\
\mathrm{C}_{\max } \text { decrease by } \\
68.3 \% \dagger\end{array}$ \\
\hline Omeprazole & 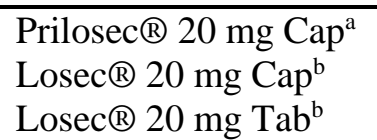 & $\begin{array}{l}\text { Major substrate of CYP2C19 } \\
\text { Minor substrate of CYP2A6, } \\
\text { CYP2C9, CYP2D6, CYP3A4 }\end{array}$ & No FE \\
\hline Lansoprazole & $\begin{array}{l}\text { Prevacid® } 30 \mathrm{mg} \\
\text { Cap }^{\text {ab }}\end{array}$ & $\begin{array}{l}\text { Major substrate of CYP2C19, } \\
\text { CYP3A4 } \\
\text { Minor substrate of CYP2C9 }\end{array}$ & $\begin{array}{l}\text { AUC decrease by } \\
50-70 \% \\
C_{\max } \text { decrease by } \\
50-70 \%\end{array}$ \\
\hline Pantoprazole $\mathrm{Na}$ & Pantoloc®40 mg Tab ${ }^{\mathrm{b}}$ & $\begin{array}{l}\text { Major substrate of CYP2C19 } \\
\text { Minor substrate of CYP2D6, } \\
\text { CYP3A4 }\end{array}$ & No FE \\
\hline $\begin{array}{l}\text { Pantoprazole } \\
\mathrm{Mg}\end{array}$ & Tecta®40 mg Tab ${ }^{\mathrm{b}}$ & $\begin{array}{l}\text { Major substrate of CYP2C19 } \\
\text { Minor substrate of CYP2D6, } \\
\text { CYP3A4 }\end{array}$ & No FE \\
\hline Rabeprazole & Pariet $^{\mathrm{TM}} / \AA 20 \mathrm{mg} \mathrm{Tab}{ }^{\mathrm{b}}$ & $\begin{array}{l}\text { Major substrate of CYP2C19, } \\
\text { CYP3A4 }\end{array}$ & No FE \\
\hline
\end{tabular}

API, Active pharmaceutical ingredient; FE, Food effect; Tab, Tablet; Cap, Capsule; SR, Sustained release; CD, Controlled Delivery; CR, Controlled release; Na, Sodium; Mg, Magnesium; ${ }^{\psi}$ Extracted from literature (36-39); ${ }^{\text {a }}$ US Reference Listed Drug (RLD) Product; ${ }^{\mathrm{b}}$ Canadian Reference Product; $\$$ As per label by FDA, $\mathrm{C}_{\max }$ decreased by $100 \%$ in the presence of food; $\uparrow$ The magnitude of decrease in the AUC and $\mathrm{C}_{\max }$ is derived from esomeprazole (NEXIUM®) $20 \mathrm{mg}$ Tab Clinical Pharmacology and Biopharmaceutics Review, NDA 207920. As per monograph, NEXIUM® may be taken with or without food. 
Table 2. Summary Food Effect in North America and India represented as Point Estimate (PE) and 95\% Confidence Intervals (CI) for $\mathrm{AUC}_{0-\mathrm{t}}$.

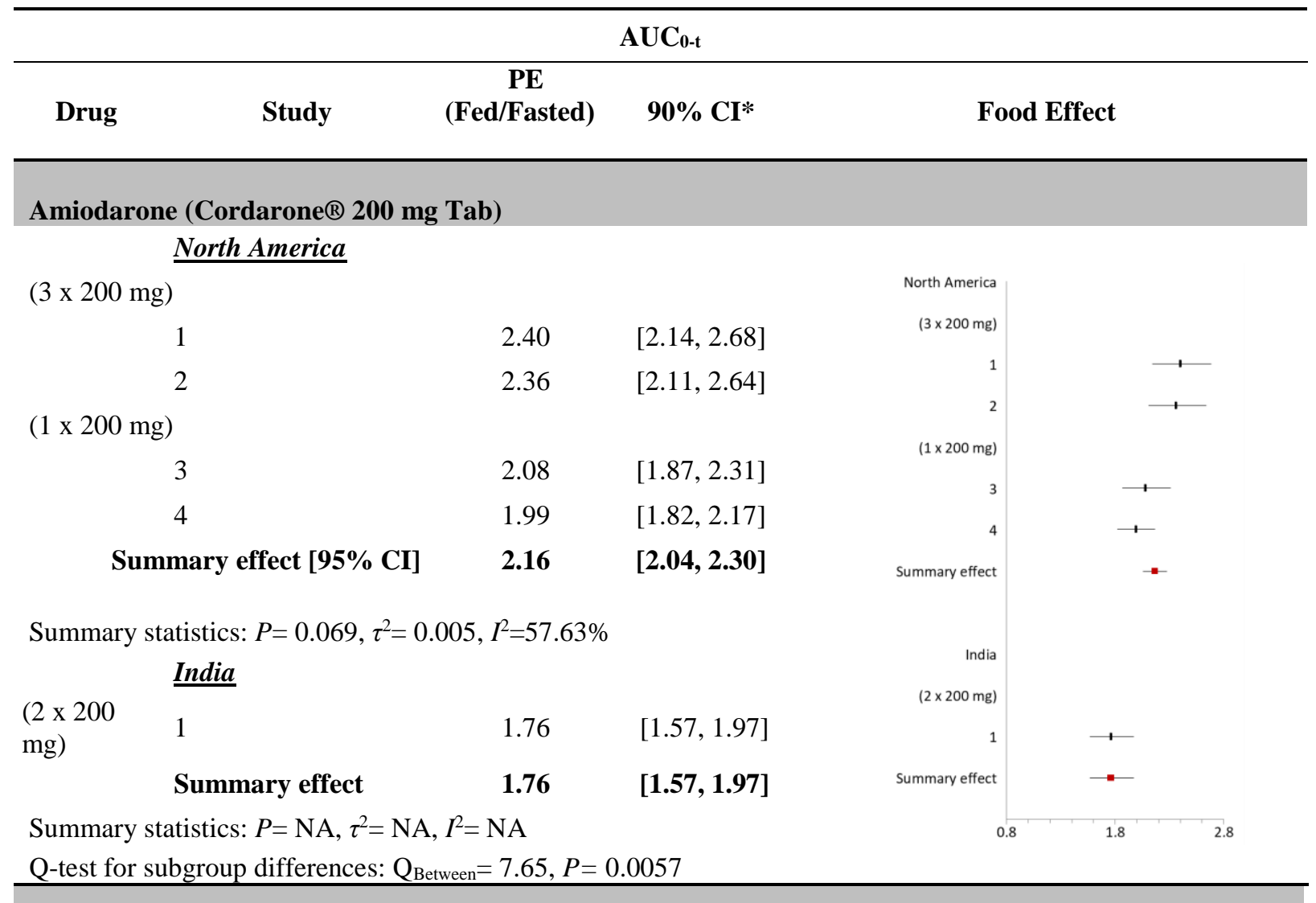

Carbamazepine (Tegretol® CR $400 \mathrm{mg}$ Tab) $(1$ x $400 \mathrm{mg})$

North America

$\begin{array}{lll}1 & 1.49 & {[1.38,1.60]} \\ 2 & 1.16 & {[1.09,1.23]}\end{array}$

Summary effect $[95 \% \mathrm{CI}] \quad 1.27 \quad[1.21,1.35]$

Summary statistics: $P<0.0001, \tau^{2}=0.029, I^{2}=94.80 \%$

\section{$\underline{\text { India }}$}

1

1.15

$[1.08,1.21]$

Summary effect

1.15

$[1.08,1.21]$

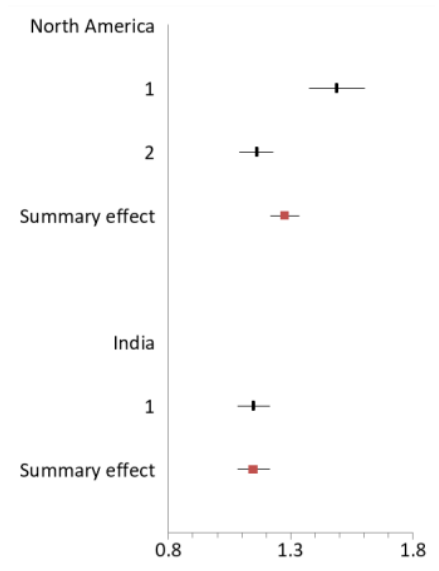

Summary statistics: $P=\mathrm{NA}, \tau^{2}=\mathrm{NA}, I^{2}=\mathrm{NA}$

Q-test for subgroup differences: $\mathrm{Q}_{\text {Between }}=5.81, P=0.01$

Table 2 continues .... 


\begin{tabular}{|c|c|c|c|}
\hline \multicolumn{4}{|l|}{ Diltiazem } \\
\hline \multicolumn{4}{|l|}{ North America } \\
\hline \multicolumn{4}{|l|}{ Cardizem $^{\circledast}$ SR 120 mg Cap $(1$ x 120 mg) } \\
\hline 1 & 1.06 & & {$[0.92,1.23]$} \\
\hline 2 & 1.27 & & {$[1.12,1.44]$} \\
\hline 3 & 1.02 & & {$[0.91,1.15]$} \\
\hline Summary effect $[95 \% \mathrm{Cl}]$ & 1.11 & & {$[1.02,1.21]$} \\
\hline \multicolumn{4}{|c|}{ Summary statistics: $P=0.09, \tau^{2}=0.008, I^{2}=58.46 \%$} \\
\hline \multicolumn{4}{|c|}{ Cardizem $^{\circledR}$ CD 360 mg Cap ( 1 x 360 mg) } \\
\hline 4 & 0.97 & & {$[0.83,1.13]$} \\
\hline 5 & 1.10 & & {$[0.95,1.27]$} \\
\hline \multicolumn{4}{|c|}{ Cardizem $^{\circledR}$ CD 300 mg Cap (1 x 300 mg) } \\
\hline 6 & 1.24 & & {$[1.03,1.50]$} \\
\hline 7 & 1.36 & & {$[1.18,1.57]$} \\
\hline 8 & 0.93 & & {$[0.81,1.07]$} \\
\hline Summary effect $[95 \% \mathrm{Cl}]$ & 1.10 & & {$[1.02,1.19]$} \\
\hline \multicolumn{4}{|c|}{ Summary statistics: $P=0.009, \tau^{2}=0.019, I^{2}=70.65 \%$} \\
\hline \multicolumn{4}{|l|}{ Tiazac $^{\circledR}$ XC 360 mg Tab (1 x 360 mg) } \\
\hline 9 & & 1.01 & {$[1.12,1.25]$} \\
\hline
\end{tabular}

Tiazac $^{\circledR}$ ER 360 mg Cap $(1 \times 360 \mathrm{mg})$

\section{North America}

10

No Food Effect

India

1

0.98

$[0.85,1.13]$

2

0.98

$[0.86,1.10]$

Summary effect $[95 \% \mathrm{Cl}]$

0.98

$[0.88,1.09]$

Summary statistics: $P=0.965, \tau^{2}=0, I^{2}=0 \%$
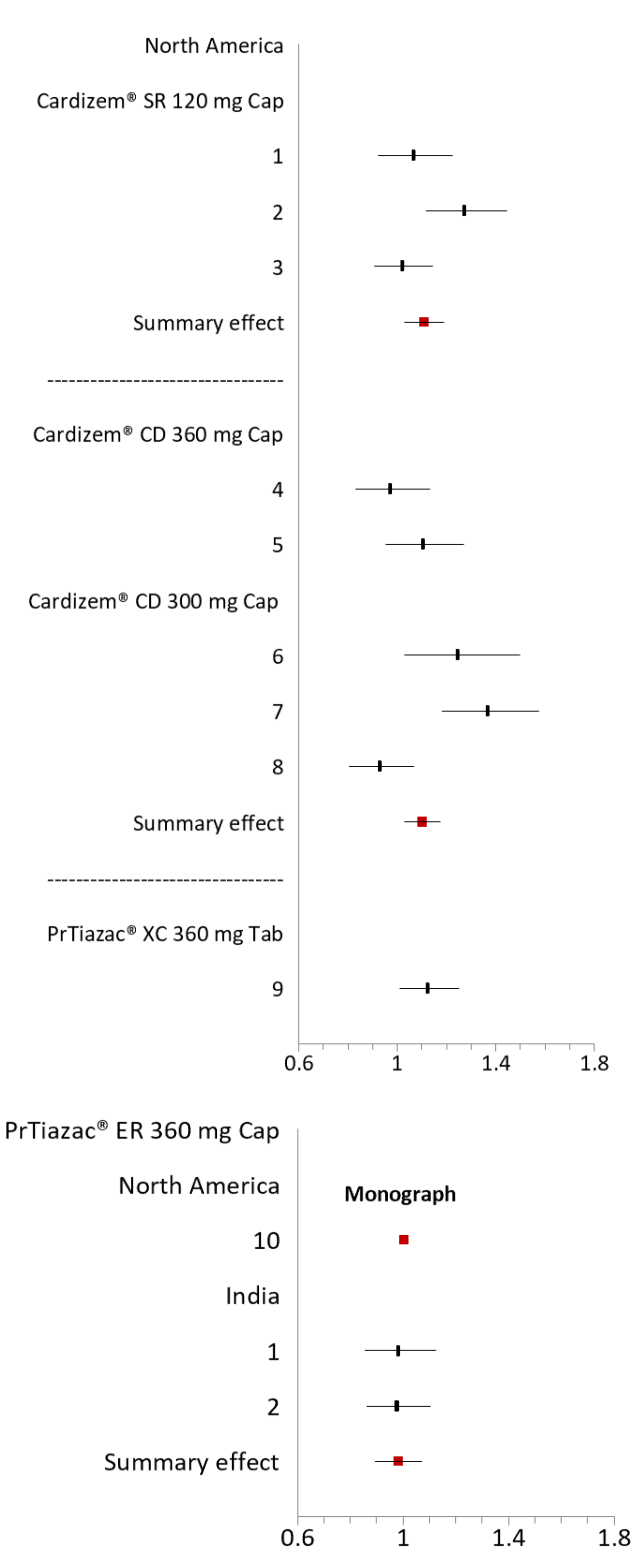

No PK bioequivalence study was available in North American population. As per monograph, the extent of diltiazem absorption was not affected by food. Therefore, (fed/fasted) PE for $A U C_{0-t}$ in this population was shown to be unity (PE=1).

Table 2 continues.... 


\section{Verapamil (Isoptin® SR $240 \mathrm{mg}$ Tab) (1 x $240 \mathrm{mg})$}

\section{North America}

1

2

Summary effect $[95 \% \mathrm{CI}]$

Summary statistics: $P=0.697, \tau^{2}=0, I^{2}=0 \%$

\section{$\underline{\text { India }}$}

Summary effect

Summary statistics: $P=\mathrm{NA}, \tau^{2}=\mathrm{NA}, I^{2}=\mathrm{NA}$
$1.03 \quad[0.91,1.17]$

$0.99 \quad[0.85,1.14]$

$1.01 \quad[0.90,1.13]$
North America

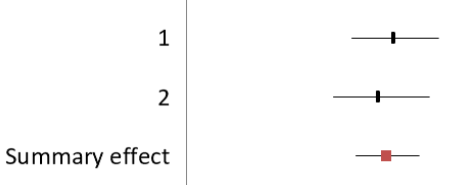

Q-test for subgroup differences: $\mathrm{Q}_{\text {Between }}=11.32, P=0.0008$

\section{$0.72 \quad[0.62,0.83]$}

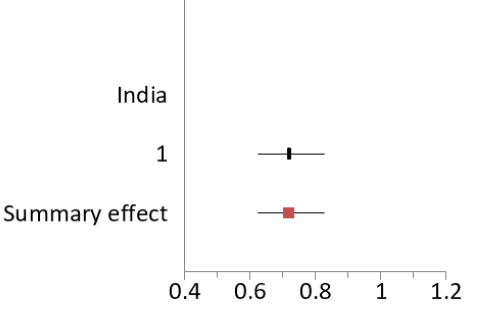

Esomeprazole (NEXIUM® $40 \mathrm{mg}$ Tab) $(1 \times 40 \mathrm{mg})$

$\begin{array}{ccc}\text { North America } & & \\ 1 & 0.48 & {[0.38,0.60]} \\ 2 & 0.51 & {[0.43,0.62]} \\ 3 & 0.56 & {[0.50,0.62]} \\ \text { Summary effect [95\% CI] } & \mathbf{0 . 5 4} & {[\mathbf{0 . 4 8 , 0 . 5 9 ]}}\end{array}$

Summary statistics: $P=0.573, \tau^{2}=0, I^{2}=0 \%$

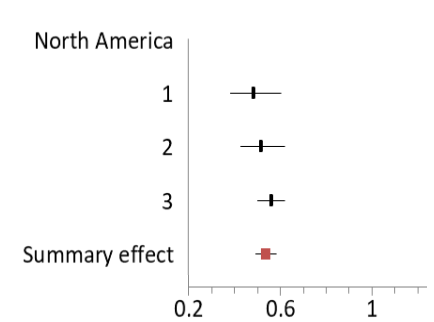

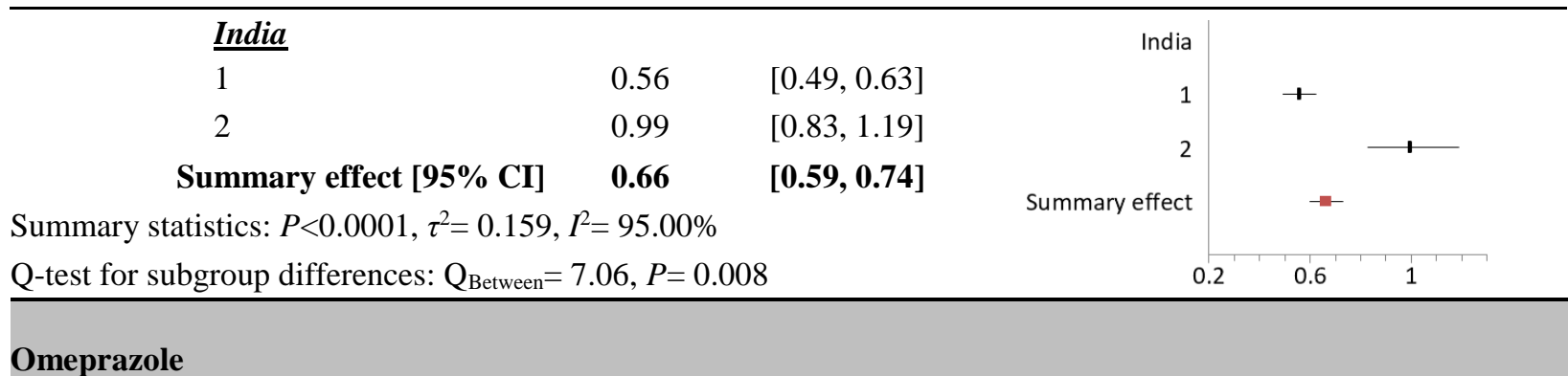

Losec ${ }^{2} 20 \mathrm{mg}$ Tab (1 x $\left.20 \mathrm{mg}\right)$

$\begin{array}{lll}\underline{\text { India }} & & \\ 1 & 1.02 & {[0.82,1.27]} \\ 2 & 1.13 & {[0.92,1.38]} \\ 3 & 1.84 & {[1.52,2.22]} \\ 4 & 0.90 & {[0.67,1.21]}\end{array}$

Summary effect $[95 \% \mathrm{CI}] \quad \mathbf{1 . 2 5} \quad[1.10,1.42]$

Summary statistics: $P=0.0004, \tau^{2}=0.087, I^{2}=83.30$

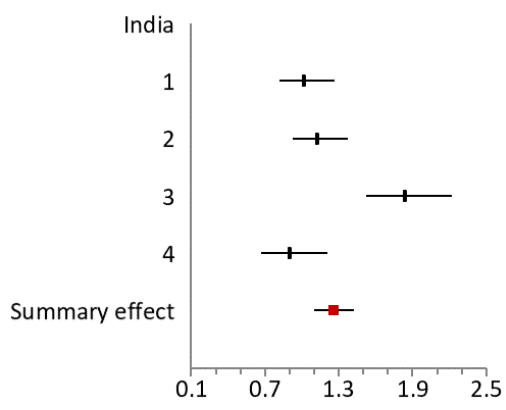

Losec ${ }^{2} 20 \mathrm{mg}$ Cap

\begin{tabular}{|c|c|c|c|}
\hline \multirow{3}{*}{$(2 \times 20 \mathrm{mg}$} & \multicolumn{2}{|l|}{$\underline{\text { North America }}$} & \\
\hline & & & \\
\hline & 1 & 0.82 & {$[0.61,1.10]$} \\
\hline \multirow{2}{*}{$(1 \times 20 \mathrm{mg}$} & 2 & 0.58 & {$[0.46 \quad 073]$} \\
\hline & Summary effect $[95 \% \mathrm{CI}]$ & 0.67 & {$[0.54,0.82]$} \\
\hline
\end{tabular}

Table 2 continues .... 
Summary statistics: $P=0.116, \tau^{2}=0.036, I^{2}=59.60 \%$

\begin{tabular}{cccc}
$(1 \times 20 \mathrm{mg})$ & \multicolumn{1}{l}{ India } \\
& & & \\
& 5 & 0.66 & {$[0.51,0.87]$} \\
& 6 & 1.42 & {$[1.10,1.82]$} \\
& Summary effect $[\mathbf{9 5 \%} \mathbf{C I}]$ & $\mathbf{1 . 0 0}$ & {$[\mathbf{0 . 8 0}, \mathbf{1 . 2 4}]$}
\end{tabular}

Summary statistics: $P=0.001, \tau^{2}=0.264, I^{2}=91.61$

Q-test for subgroup differences: $\mathrm{Q}_{\text {Between }}=6.82, P=0.009$

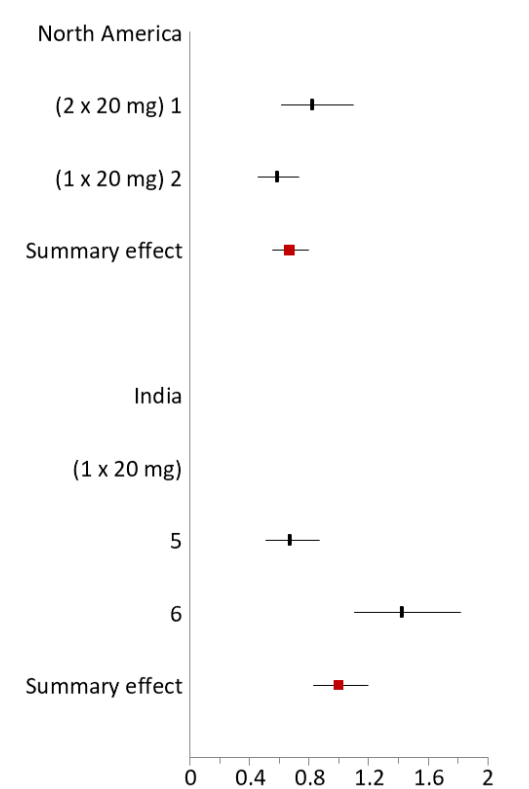

$\begin{array}{llllll}0 & 0.4 & 0.8 & 1.2 & 1.6 & 2\end{array}$

Lansoprazole (Prevacid® 30 mg Cap ) (1 x 30 mg)

\section{North America}

\begin{tabular}{lll}
\hline 1 & 0.21 & {$[0.17,0.27]$} \\
Summary effect & $\mathbf{0 . 2 1}$ & {$[\mathbf{0 . 1 7}, \mathbf{0 . 2 7}]$}
\end{tabular}

Summary statistics: $P=\mathrm{NA}, \tau^{2}=\mathrm{NA}, I^{2}=\mathrm{NA}$

$\begin{array}{ccc}\frac{\text { India }}{1} & 0.27 & {[0.23,0.32]} \\ 2 & 0.48 & {[0.41,0.56]} \\ 3 & 0.15 & {[0.12,0.19]} \\ 4 & 0.27 & {[0.22,0.33]} \\ \text { Summary effect [95\% CI] } & \mathbf{0 . 3 0} & {[\mathbf{0 . 2 6}, \mathbf{0 . 3 3}]}\end{array}$

Summary statistics: $P<0.0001, \tau^{2}=0.212, I^{2}=94.49 \%$

Q-test for subgroup differences: $Q_{\text {Between }}=4.04, P=0.04$

Pantoprazole Na (Pantoloc $® 40$ mg Tab) (1 x 40 mg)

\section{North America}

$\begin{array}{ccc}1 & 0.81 & {[0.69,0.96]} \\ 2 & 0.86 & {[0.73,1.01]} \\ \text { Summary effect [95\% CI] } & \mathbf{0 . 8 4} & {[\mathbf{0 . 7 3}, \mathbf{0 . 9 6}]}\end{array}$

Summary statistics: $P=0.684, \tau^{2}=0, I^{2}=0 \%$

\section{$\underline{\text { India }}$}

$\begin{array}{lll}1 & 1.06 & {[0.87,1.29]} \\ 2 & 0.74 & {[0.62,0.89]} \\ 3 & 0.67 & {[0.53,0.83]} \\ 4 & 0.72 & {[0.64,0.82]}\end{array}$




$\begin{array}{lll}5 & 0.92 & {[0.78,1.08]} \\ 6 & 0.74 & {[0.62,0.89]} \\ \text { mmary effect [95\% CI] } & \mathbf{0 . 7 9} & {[\mathbf{0 . 7 3 , 0 . 8 6}]}\end{array}$

Summary effect $[95 \% \mathrm{CI}] \quad 0.79 \quad[0.73,0.86]$

Summary statistics: $P=0.035, \tau^{2}=0.015, I^{2}=58.26 \%$

Q-test for subgroup differences: $\mathrm{Q}_{\text {Between }}=0.45, P=0.504$

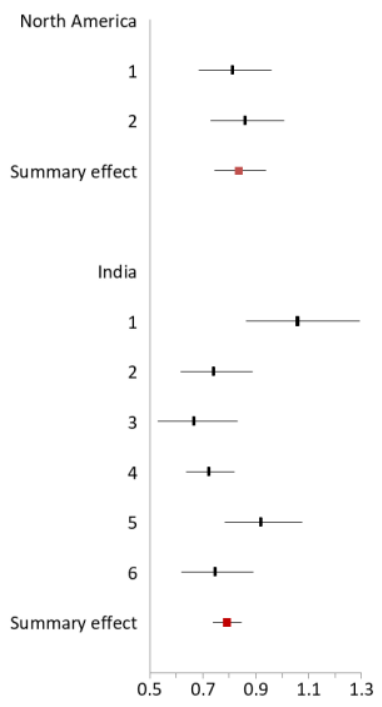

Pantoprazole Mg (Tecta ${ }^{\circledR} 40 \mathrm{mg}$ Tab) $(1$ x $40 \mathrm{mg})$

North America

1

0.77

$[0.68,0.88]$

$\underline{\text { India }}$

1

1.17

$[0.93,1.48]$

North America

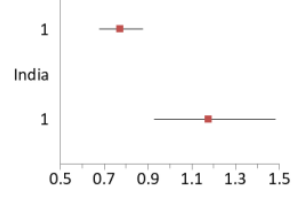

Rabeprazole (Pariet ${ }^{\mathrm{TM}} /$ Pariet ${ }^{\circledR} 20 \mathrm{mg}$ Tab) $(1$ x 20 mg)

North America

1

0.78

$[0.69,0.89]$

2

0.82

$[0.72,0.94]$

Summary effect $[95 \% \mathrm{CI}] \quad \mathbf{0 . 8 0}$

Summary statistics: $P=0.64, \tau^{2}=0, I^{2}=0 \%$

$[0.72,0.89]$

India

1

0.90

$[0.78,1.04]$

2

1.15

$[1.02,1.30]$

3

1.51

$[1.34,1.70]$

Summary effect $[95 \% \mathrm{CI}]$

1.19

$[1.09,1.30]$

Summary statistics: $P<0.0001, \tau^{2}=0.056, I^{2}=90.60 \%$

Q-test for subgroup differences: $\mathrm{Q}_{\text {Between }}=31.63, P=0.00001$

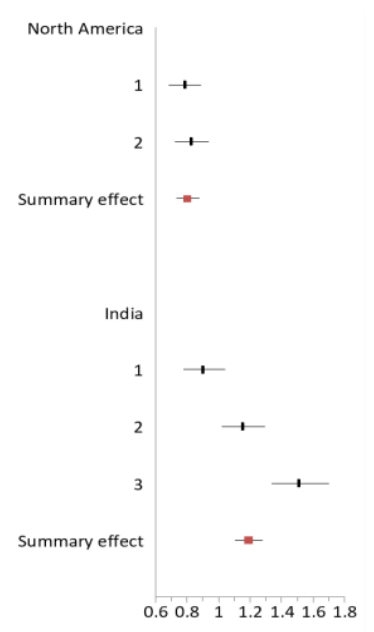

The vertical straight lines denote the GMRs of $\mathrm{AUC}_{0-\mathrm{t}}$ (fed/fasted) for each study and the lines on either side the $90 \%$ confidence intervals. The summary effect is represented by a red square and the lines on either side standing for point estimate and $95 \%$ confidence intervals, respectively, on the bottom line of each population. The $P$-value in summary statistics of each population corresponds to the variance of studies within that subgroup population and demonstrates whether the variance within subgroup is statistically significant. The $P$-value corresponding to Q Between on the bottom line of forest plot for each drug demonstrates whether the summary effect is the same for studies in North America as for the studies in India. PE, Point Estimate; GMR, Geometric Mean Ratio; CI, Confidence interval; NA, Not Applicable; Na, Sodium; Mg, Magnesium; $\tau^{2}$, measure of heterogeneity (between-studies variance); $I^{2}$, measure of heterogeneity (degree of inconsistency in \%); *95\% CI for summary food effect of each population. 
Table 3. Summary Food Effect in North America and India represented as Point Estimate (PE) and 95\% Confidence Intrvals (CI) for $\mathrm{C}_{\max }$

\begin{tabular}{|c|c|c|c|c|}
\hline Drug & Study & $\begin{array}{c}\text { PE } \\
\text { (Fed/Fasted) }\end{array}$ & $90 \% \mathrm{CI}^{*}$ & Food Effect \\
\hline
\end{tabular}

\section{North America}

(3 x $200 \mathrm{mg})$

$\begin{array}{lll}3.77 & {[3.46,4.11]} \\ 2 & 3.68 & {[3.22,4.22]}\end{array}$

(1 x $200 \mathrm{mg})$

$\begin{array}{llc}3 & 2.87 & {[2.59,3.18]} \\ 4 & 2.66 & {[2.41,2.93]} \\ \text { Summary effect [95\% CI] } & \mathbf{3 . 2 0} & {[\mathbf{3 . 0 2}, \mathbf{3 . 4 0}]}\end{array}$

Summary statistics: $P<0.0001, \tau^{2}=0.03, I^{2}=88.64 \%$

$(2 \times 200 \mathrm{mg}) \frac{\text { India }}{1}$

$2.48 \quad[2.17,2.83]$

Summary effect

2.48

Summary statistics: $P=\mathrm{NA}, \tau^{2}=\mathrm{NA}, I^{2}=\mathrm{NA}$

$[2.17,2.83]$

Q-test for subgroup differences: $Q_{\text {Between }}=9.27, P=0.0023$

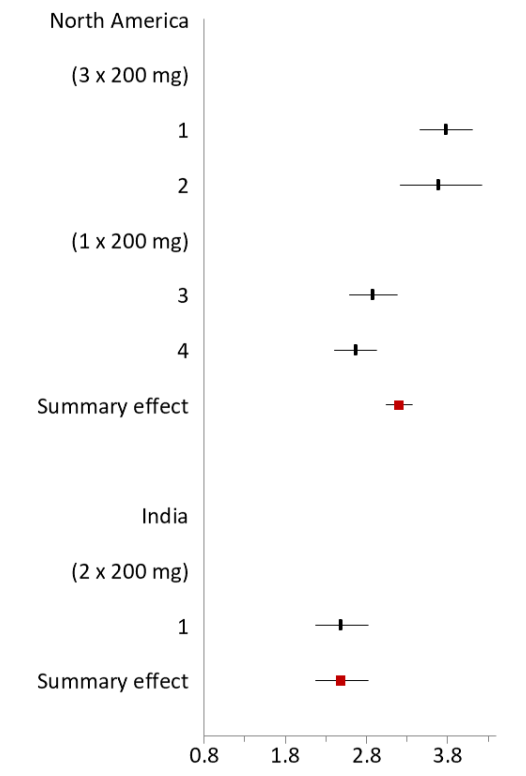

\section{Carbamazepine (Tegretol® CR $400 \mathrm{mg}$ Tab) $(1 \times 400 \mathrm{mg})$}

$\begin{array}{lll}\text { North America } & & \\ 1 & 1.46 & {[1.36,1.57]} \\ 2 & 1.21 & {[1.16,1.27]} \\ \text { Summary effect [95\% CI] } & \mathbf{1 . 2 9} & {[\mathbf{1 . 2 3}, \mathbf{1 . 3 5}]}\end{array}$

Summary statistics: $P=0.0003, \tau^{2}=0.016, I^{2}=92.31 \%$

$\underline{\text { India }}$

1

1.12

$[1.07,1.18]$

Summary effect

$1.12 \quad[1.07,1.18]$

Summary statistics: $P=\mathrm{NA}, \tau^{2}=\mathrm{NA}, I^{2}=\mathrm{NA}$

Q-test for subgroup differences: $\mathrm{Q}_{\text {Between }}=11.86, P=0.0006$

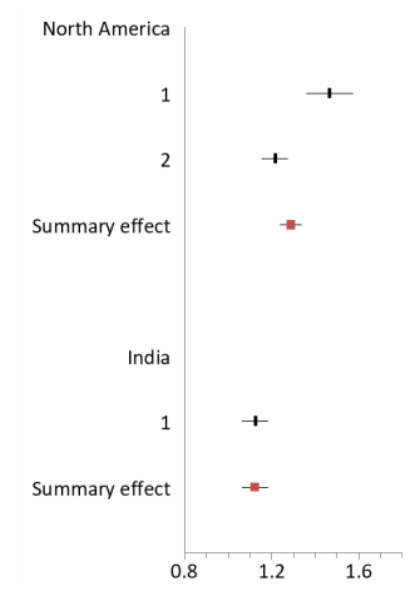

\section{Diltiazem}

\section{North America}

Cardizem® SR 120 mg Cap (1 x 120 mg)

$\begin{array}{lll}1 & 1.12 & {[0.97,1.29]} \\ 2 & 1.33 & {[1.17,1.51]}\end{array}$

Table 3 continues .... 


\section{3 Summary effect $[95 \% \mathrm{CI}]$ \\ 0.95 \\ 1.10 \\ $[0.85,1.06]$ \\ $[1.01,1.20]$}

Summary statistics: $P=0.003, \tau^{2}=0.026, I^{2}=82.36 \%$

Cardizem® CD $360 \mathrm{mg}$ Cap (1 x $360 \mathrm{mg})$

$\begin{array}{lll}4 & 1.04 & {[0.87,1.25]} \\ 5 & 1.12 & {[0.99,1.26]} \\ 6 & 1.10 & {[0.97,1.25]} \\ 7 & 1.24 & {[1.06,1.44]} \\ 8 & 1.07 & {[0.93,1.23]}\end{array}$

Cardizem® CD 300 mg Cap (1 x 300 mg)

$\begin{array}{ll}9 & 1.03 \\ 10 & 1.34 \\ 11 & 0.80\end{array}$

Summary effect $[95 \%$ CI $] \quad 1.09$

$[0.71,0.92]$

$[1.03,1.15]$

Summary statistics: $P=0.0008, \tau^{2}=0.018, I^{2}=71.98 \%$

PrTiazac® XC 360 mg Tab (1 x 360 mg)

12

1.04

$[0.93,1.17]$

PrTiazac® ER 360 mg Cap (1 x 360 mg)

North America

13

Slightly $>1$

$\underline{\text { India }}$

$\begin{array}{ll}1 & 1.36 \\ 2 & 1.40\end{array}$

Summary effect $\left[\begin{array}{ll}95 \% & C I\end{array}\right]$

Summary statistics: $P=0.728, \tau^{2}=0, I^{2}=0 \%$

No information regarding the effect of food on $\mathrm{C}_{\max }$ was available in the North American population. As per monograph, in the presence of food $t_{\max }$ occurred slightly earlier. Since earlier $t_{\max }$ appears in parallel with increased $\mathrm{C}_{\max }$, the PE (fed/fasted) for $\mathrm{C}_{\max }$ was assumed to be slightly larger than unity.

\section{Verapamil (Isoptin® SR 240 mg Tab) $(1$ x 240 mg)}

$\begin{array}{lll}\text { North America } & 0.76 & {[0.66,0.88]} \\ 1 & 0.64 & {[0.54,0.77]} \\ 2 & & {[\mathbf{0 . 6 2}, \mathbf{0 . 8 1}]}\end{array}$

Summary statistics: $P=0.224, \tau^{2}=0.005, I^{2}=32.50 \%$

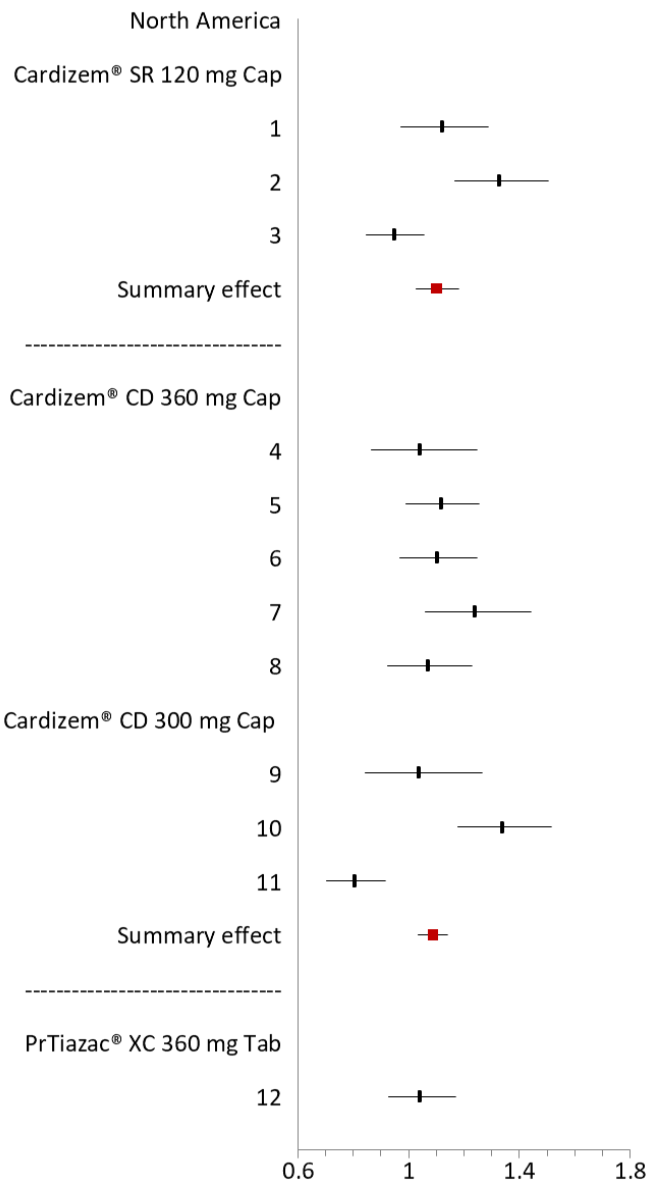

$\operatorname{PrTiazac}^{\oplus} \mathrm{ER} 360 \mathrm{mg}$ Cap

North America

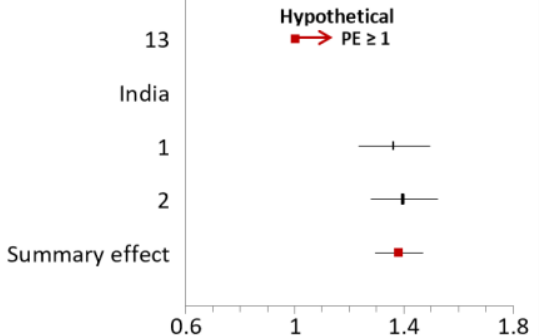

$[1.28,1.49]$
$[0.62,0.81]$$$
0 \%
$$

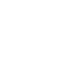




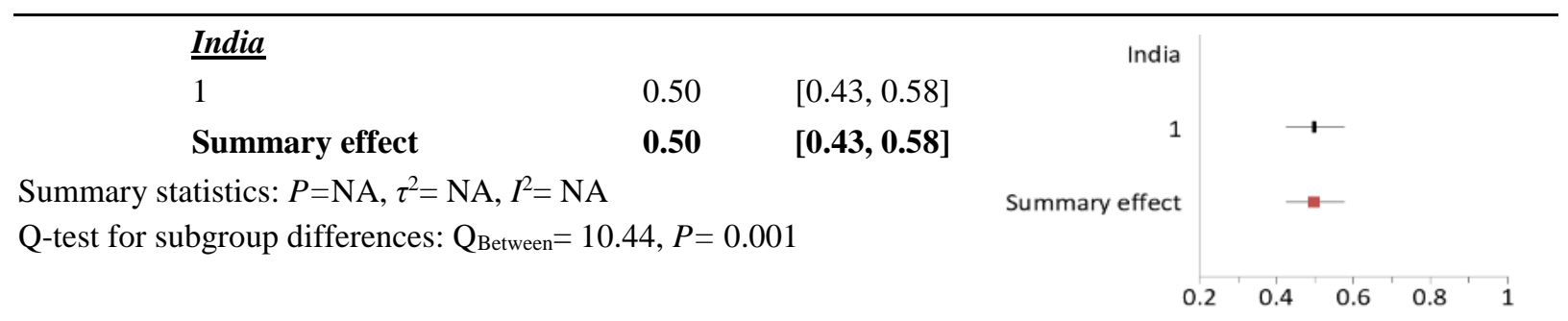

Esomeprazole (NEXIUM® $40 \mathrm{mg}$ Tab) (1 x $40 \mathrm{mg}$ )

\begin{tabular}{lll} 
North America & & \\
\hline 1 & 0.41 & {$[0.34,0.49]$} \\
2 & 0.34 & {$[0.29,0.40]$} \\
3 & 0.42 & {$[0.38,0.46]$} \\
Summary effect [95\% CI] & $\mathbf{0 . 4 0}$ & {$[\mathbf{0 . 3 7}, \mathbf{0 . 4 4}]$}
\end{tabular}

Summary statistics: $P=0.171, \tau^{2}=0.006, I^{2}=43.42$

$\begin{array}{lll}\underline{\text { India }} & & \\ 1 & 0.42 & {[0.38,0.46]} \\ 2 & 0.67 & {[0.59,0.77]} \\ \text { Summary effect [95\% CI] } & \mathbf{0 . 4 9} & {[\mathbf{0 . 4 4}, \mathbf{0 . 5 4}]}\end{array}$

Summary statistics: $P<0.0001, \tau^{2}=0.112, I^{2}=95.68 \%$

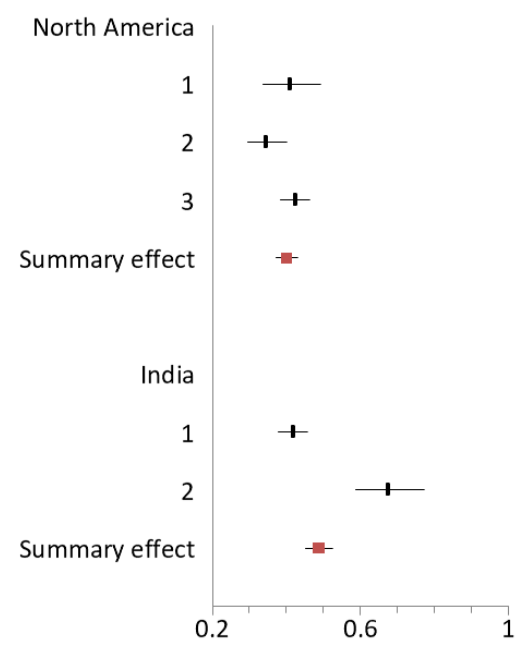

Q-test for subgroup differences: $\mathrm{Q}_{\text {Between }}=9.23, P=0.0024$

\section{Omeprazole}

Losec ${ }^{\circledR} 20 \mathrm{mg}$ Tab (1 x $\left.20 \mathrm{mg}\right)$

$\begin{array}{lll}\underline{\underline{\text { India }}} & & \\ 1 & 1.32 & {[1.15,1.52]} \\ 2 & 1.10 & {[0.97,1.25]} \\ 3 & 1.74 & {[1.55,1.95]} \\ 4 & 0.97 & {[0.80,1.17]} \\ \text { Summary effect [95\% CI] } & \mathbf{1 . 3 3} & {[\mathbf{1 . 2 2}, \mathbf{1 . 4 4}]}\end{array}$

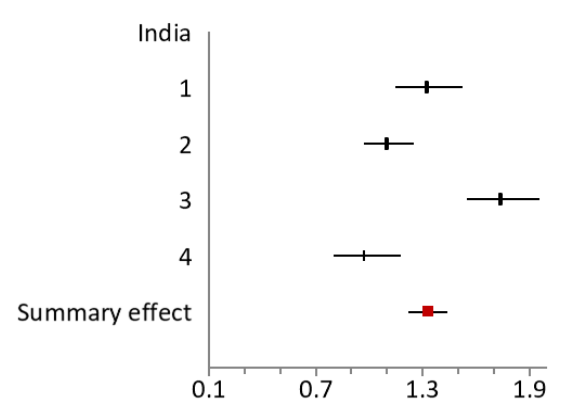

Summary statistics: $P<0.00001, \tau^{2}=0.059, I^{2}=89.37$ 
Losec $® 20$ mg Cap

\begin{tabular}{|c|c|c|c|}
\hline$(2 \times 20 \mathrm{mg})$ & \multicolumn{3}{|l|}{ North America } \\
\hline & & 0.65 & {$[0.53,0.81]$} \\
\hline \multicolumn{4}{|l|}{$(1 \times 20 \mathrm{mg})$} \\
\hline & & 0.33 & {$[0.28,0.38]$} \\
\hline & Summary effect $[95 \% \mathrm{CI}]$ & 0.42 & {$[0.36,0.48$} \\
\hline \multirow{2}{*}{\multicolumn{4}{|c|}{$\begin{array}{l}\text { Summary statistics: } P<0.0001, \tau^{2}=0.219, I^{2}=94.83 \% \\
(1 \mathrm{x} 20 \mathrm{mg}) \underline{\text { India }}\end{array}$}} \\
\hline & & & \\
\hline 5 & & 0.49 & {$[0.41,0.57]$} \\
\hline & 6 & 0.84 & {$[0.69,1.02]$} \\
\hline & Summary effect $[95 \%$ CI $]$ & 0.61 & {$[0.53,0.71]$} \\
\hline
\end{tabular}

Summary statistics: $P=0.0003, \tau^{2}=0.139, I^{2}=92.39$

Q-test for subgroup differences: $\mathrm{Q}_{\text {Between }}=13.74, P=0.0002$

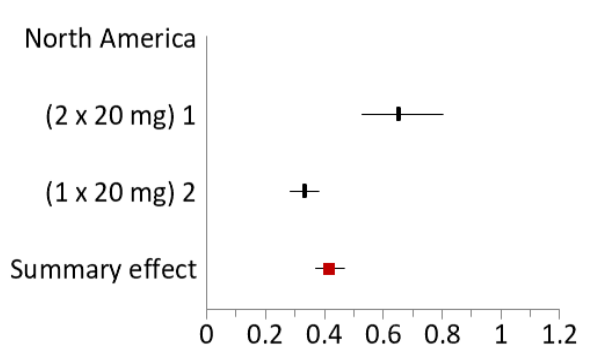

Lansoprazole (Prevacid® 30 mg Cap ) (1 x 30 mg)

$\begin{array}{lll}\text { North America } & & \\ 1 & 0.16 & {[0.14,0.18]} \\ \text { Summary effect } & \mathbf{0 . 1 6} & {[\mathbf{0 . 1 4}, \mathbf{0 . 1 8}]}\end{array}$

Summary statistics: $P=\mathrm{NA}, \tau^{2}=\mathrm{NA}, I^{2}=\mathrm{NA}$

$\begin{array}{lll}\underline{\text { India }} & & \\ 1 & 0.22 & {[0.19,0.26]} \\ 2 & 0.36 & {[0.33,0.40]} \\ 3 & 0.16 & {[0.14,0.19]} \\ 4 & 0.23 & {[0.19,0.27]} \\ \text { Summary effect [95\% CI] } & \mathbf{0 . 2 6} & {[\mathbf{0 . 2 4}, \mathbf{0 . 2 8}]}\end{array}$

Summary statistics: $P<0.0001, \tau^{2}=0.129, I^{2}=94.71 \%$

Q-test for subgroup differences: $\mathrm{Q}_{\text {Between }}=43.03, P<0.0001$

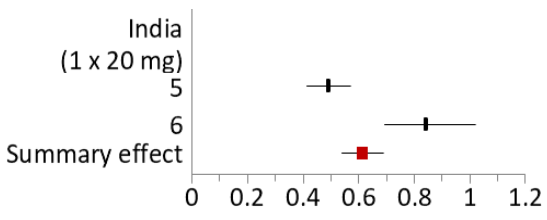

Pantoprazole Na (Pantoloc ${ }^{\circ} 40$ mg Tab) (1 x 40 mg)

\section{$\underline{\text { North America }}$}

$\begin{array}{lll}1 & 0.72 & {[0.64,0.80]} \\ 2 & 0.68 & {[0.64,0.74]}\end{array}$

Summary effect $[95 \% \mathrm{CI}] \quad 0.70 \quad[0.65,0.75]$

Summary statistics: $P=0.543, \tau^{2}=0, I^{2}=0 \%$

\section{India}

1

$0.86 \quad[0.79,0.93]$

Table 3 continues .... 


$\begin{array}{lll}2 & 0.82 & {[0.74,0.91]} \\ 3 & 0.81 & {[0.74,0.89]} \\ 4 & 0.87 & {[0.80,0.95]} \\ 5 & 0.79 & {[0.74,0.84]} \\ 6 & 0.75 & {[0.70,0.80]} \\ \text { Summary effect [95\% CI] } & \mathbf{0 . 8 1} & {[\mathbf{0 . 7 8 , 0 . 8 4}]}\end{array}$

Summary statistics: $P=0.204, \tau^{2}=0.001, I^{2}=30.89 \%$

Q-test for subgroup differences: $\mathrm{Q}_{\text {Between }}=12.66, P<0.0004$

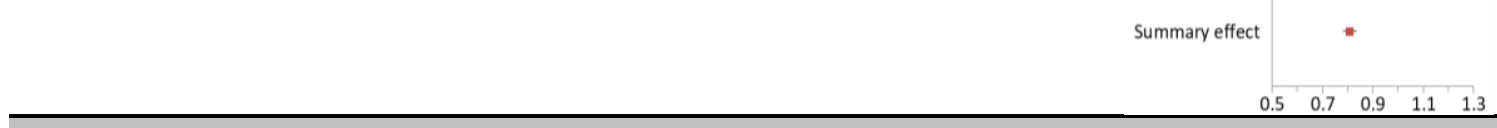

Pantoprazole Mg (Tecta ${ }^{\circledR} 40 \mathrm{mg}$ Tab) $(1$ x $40 \mathrm{mg})$

\section{North America}

1

0.89

$[0.82,0.97]$

$\underline{\text { India }}$

1

1.10

$[0.97,1.25]$

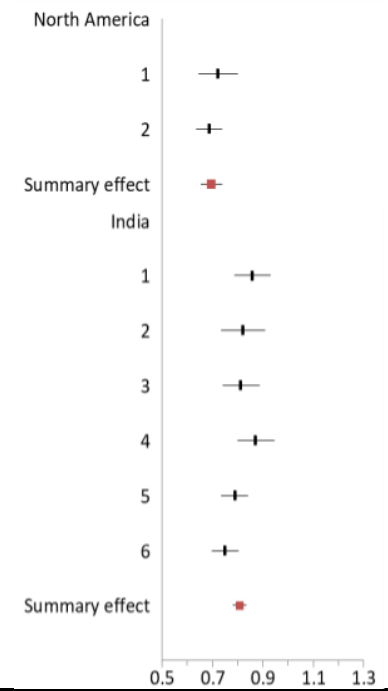

\begin{tabular}{|c|c|c|}
\hline \multicolumn{3}{|l|}{ North America } \\
\hline 1 & 0.67 & {$[0.60,0.76]$} \\
\hline 2 & 0.60 & {$[0.54,0.67]$} \\
\hline Summary effect $[95 \%$ CI $]$ & 0.63 & {$[0.58,0.70]$} \\
\hline
\end{tabular}

Summary statistics: $\mathrm{P}=0.261, \tau^{2}=0.001, I^{2}=20.88$

$\underline{\text { India }}$

1

0.96

$[0.85,1.09]$

2

1.67

$[1.50,1.87]$

3

1.77

$[1.59,1.96]$

Summary effect $[95 \% \mathrm{CI}]$

1.47

$[1.36,1.59]$

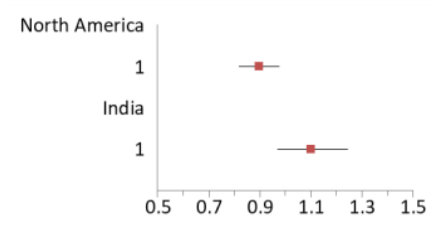

Summary statistics: $P<0.00001, \tau^{2}=0.098, I^{2}=95.44 \%$

Q-test for subgroup differences: $\mathrm{Q}_{\text {Between }}=184.18, P=0.00001$

The vertical straight lines denote the GMRs of $\mathrm{C}_{\max }$ (fed/fasted) for each study and the lines on either side the $90 \%$ confidence intervals. The summary effect is represented by a red square and the lines on either side standing for point estimate and 95\% confidence intervals, respectively, on the bottom line of each population. The $P$-value in summary statistics of each population corresponds to the variance of studies within that subgroup population and demonstrates whether the variance within subgroup is statistically significant. The $P$-value corresponding to $\mathrm{Q}_{\text {Between }}$ on the bottom line of forest plot for each drug demonstrates whether the summary effect is the same for studies in North America as for the studies in India. PE, Point Estimate; GMR, Geometric Mean Ratio; CI, Confidence interval; NA, Not Applicable; Na, Sodium; Mg, Magnesium; $\tau 2$, measure of heterogeneity (between-studies variance); $\mathrm{I}^{2}$; measure of heterogeneity (degree of inconsistency in $\%$ ); $* 95 \% \mathrm{CI}$ for summary food effect of each population. 
Table 4. Heterogeneity $\left(I^{2} \%\right)$ of Food Effect from clinical PK bioequivalence studies conducted in North American and Indian populations

\begin{tabular}{|c|c|c|c|c|c|c|c|}
\hline \multirow{3}{*}{$\begin{array}{l}\text { API } \\
\text { Amiodarone }\end{array}$} & \multirow{3}{*}{$\begin{array}{l}\mathbf{n}_{\mathrm{NA}} \\
4 \\
4\end{array}$} & \multirow{3}{*}{$\begin{array}{c}\mathbf{n}_{\text {India }} \\
1 \\
1\end{array}$} & \multirow{3}{*}{$\begin{array}{c}\text { Drug Product } \\
\text { Cordarone }{ }^{2} 200 \mathrm{mg} \mathrm{Tab}\end{array}$} & \multicolumn{2}{|c|}{$\begin{array}{l}I^{2} \text { (North } \\
\text { American) }\end{array}$} & \multicolumn{2}{|c|}{$I^{2}$ (Indian) } \\
\hline & & & & $\mathrm{AUC}_{0-\mathrm{t}}$ & $\mathrm{C}_{\max }$ & $\mathrm{AUC}_{0-\mathrm{t}}$ & $\mathrm{C}_{\max }$ \\
\hline & & & & 57.63 & 88.64 & - & - \\
\hline Carbamazepine & 2 & 1 & Tegretol® CR 400 mg Tab & 94.8 & 92.31 & - & - \\
\hline \multirow[t]{3}{*}{ Diltiazem } & 3 & - & Cardizem® SR 120 mg Cap & 58.46 & 82.36 & - & - \\
\hline & 5 & - & $\begin{array}{l}\text { Cardizem }{ }^{\circledR} \text { CD } 300 \& 360 \\
\text { mg Cap }\end{array}$ & 70.65 & 71.98 & - & - \\
\hline & 1 & 2 & Tiazac ® ER 360 mg Cap & - & - & 0 & 0 \\
\hline Verapamil & 2 & 1 & Isoptin® SR $240 \mathrm{mg}$ Tab & 0 & 32.5 & - & - \\
\hline Esomeprazole $\psi$ & 3 & 2 & Nexium ${ }^{\circledR}$ PR 40 mg Tab & 0 & 43.42 & 95 & 95.78 \\
\hline \multirow[t]{2}{*}{ Omeprazole ${ }^{\psi}$} & 2 & 2 & Losec $® 20$ mg Cap & 59.6 & 94.83 & 91.61 & 92.39 \\
\hline & 4 & - & Losec $® 20$ mg Tab & - & - & 83.3 & 89.37 \\
\hline Lansoprazole & 1 & 4 & Prevacid® 30 mg Cap & - & - & 94.49 & 94.71 \\
\hline $\begin{array}{l}\text { Pantoprazole } \\
\mathrm{Na}^{\psi}\end{array}$ & 2 & 6 & Pantoloc®40 mg Tab & 0 & 0 & 58.26 & 30.89 \\
\hline Rabeprazole $\psi$ & 2 & 3 & $\operatorname{Pariet}^{\mathrm{TM}} / \circledR 20$ mg Tab & 0 & 20.88 & 90.6 & 95.44 \\
\hline \multirow{3}{*}{\multicolumn{4}{|c|}{$\begin{array}{l}\text { Common products with High } \\
\text { heterogeneity }{ }^{\psi *} \\
\text { Total number of common products }{ }^{\psi} \\
\text { \% of Common products with high heterogeneity }\left(I^{2} \geq\right. \\
\mathbf{7 5 \%})^{\psi}\end{array}$}} & 0 & 1 & 3 & 3 \\
\hline & & & & 4 & 4 & 4 & 4 \\
\hline & & & & $0 \%$ & $25 \%$ & $75 \%$ & $75 \%$ \\
\hline \multicolumn{4}{|c|}{ All products with High heterogeneity* } & 1 & 4 & 5 & 5 \\
\hline \multirow{2}{*}{\multicolumn{4}{|c|}{ Total number of products }} & 9 & 9 & 7 & 7 \\
\hline & & & & \multicolumn{4}{|c|}{$\%$ of all products with high heterogeneity $\left(I^{2} \geq 75 \%\right)$} \\
\hline
\end{tabular}

$* I^{2}$ values $\geq 75 \%$ are considered as high heterogeneity, indicating that most of the observed variance is due to the real difference in underlying true effects (food effect) between studies rather than random error.

${ }^{\psi}$ Common products represents the products with available heterogeneity data in both populations $\mathrm{n}_{\mathrm{NA}}$; Number of ANDSs in North American populations; $\mathrm{n}_{\text {India }}$, Number of ANDSs in Indian populations 
Table 5. Summary statistics of esomeprazole food effect using all available ANDS studies with Nexium ${ }^{\circledR}$ 20 and $40 \mathrm{mg}$ tablets

\begin{tabular}{cccc}
\hline AUC $_{\text {0-t }}$ & PE (Fed/Fasted) & 90\% CI* \\
NEXIUM® & $\begin{array}{c}\text { North America } \\
\text { mg Tab }(1 \times 40 \mathrm{mg})\end{array}$ & \\
1 & 0.48 & {$[0.38,0.60]$} \\
2 & 0.51 & {$[0.43,0.62]$} \\
3 & 0.56 & {$[0.50,0.62]$}
\end{tabular}

NEXIUM® $20 \mathrm{mg}$ Tab $(1 \times 20 \mathrm{mg})$

$\begin{array}{lll}4 & 0.60 & {[0.52,0.68]} \\ \text { effect }[95 \% \mathbf{C I}] & \mathbf{0 . 5 5} & {[\mathbf{0 . 5 1}, \mathbf{0 . 6 0}]}\end{array}$

Summary effect $[95 \% \mathrm{CI}] \quad 0.55 \quad[0.51,0.60]$

Summary statistics: $P=0.502, \tau^{2}=0, I^{2}=0 \%$

\section{$\underline{\text { India }}$}

NEXIUM® 40 mg Tab (1 x 40 mg)

$\begin{array}{ccc}1 & 0.56 & {[0.49,0.63]} \\ 2 & 0.99 & {[0.83,1.19]} \\ \text { effect }[\mathbf{9 5 \%} \mathbf{C I}] & \mathbf{0 . 6 6} & {[\mathbf{0 . 5 9}, \mathbf{0 . 7 4}]}\end{array}$

Summary effect $[95 \%$ CI $] \quad 0.66 \quad[0.59,0.74]$

Summary statistics: $P<0.0001, \tau^{2}=0.159, I^{2}=95.00 \%$

Q-test for subgroup differences: $\mathrm{Q}_{\text {Between }}=5.85, P=0.016$

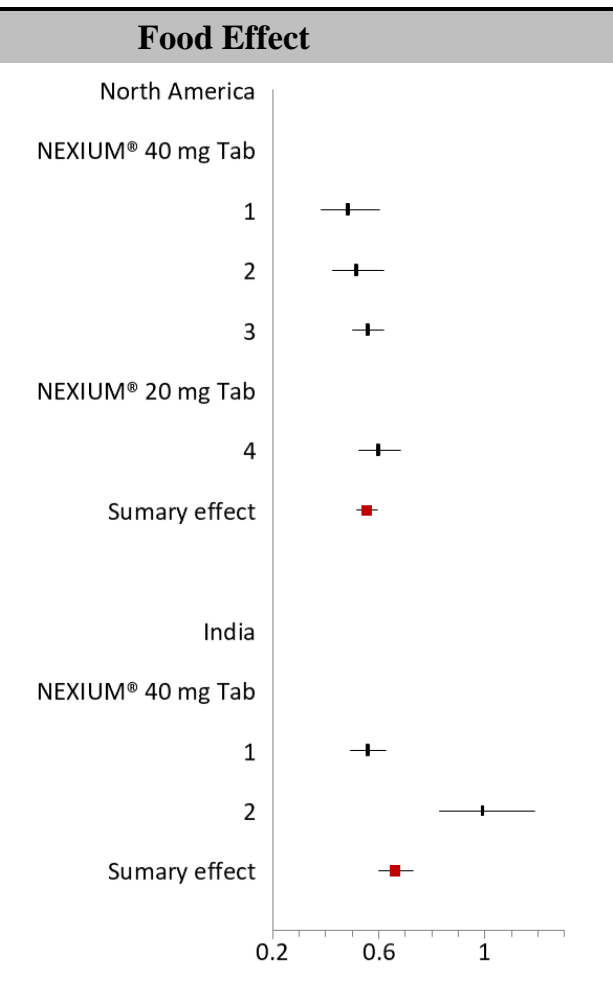

\begin{tabular}{|c|c|c|}
\hline $\mathrm{C}_{\max }$ & PE (Fed/Fasted) & $90 \% \mathrm{CI} *$ \\
\hline \multicolumn{3}{|c|}{ North America } \\
\hline \multicolumn{3}{|c|}{ NEXIUM® 40 mg Tab (1 x 40 mg) } \\
\hline 1 & 0.41 & {$[0.34,0.49]$} \\
\hline 2 & 0.34 & {$[0.29,0.40]$} \\
\hline 3 & 0.42 & {$[0.38,0.46]$} \\
\hline
\end{tabular}

NEXIUM® $20 \mathrm{mg}$ Tab $(1 \times 20 \mathrm{mg})$

$\begin{array}{ccc}4 & 0.45 & {[0.41,0.50]} \\ \text { Summary effect }[\mathbf{9 5 \%} \text { CI }] & \mathbf{0 . 4 2} & {[\mathbf{0 . 3 9}, \mathbf{0 . 4 5}]}\end{array}$

Summary statistics: $P=0.115, \tau^{2}=0.006, I^{2}=49.42 \%$

India

NEXIUM® $40 \overline{\mathrm{mg} \text { Tab }}(1 \times 40 \mathrm{mg})$

Food Effect

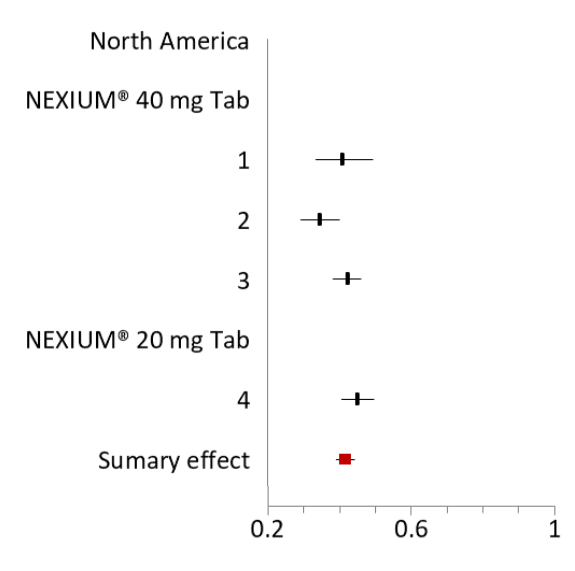
1
0.42
$[0.38,0.46]$
2
0.67
$[0.59,0.77]$
Summary effect $[95 \% \mathrm{CI}]$
0.49
$[0.44,0.54]$

Summary statistics: $P<0.0001, \tau^{2}=0.112, I^{2}=95.68 \%$

Q-test for subgroup differences: $\mathrm{Q}_{\text {Between }}=6.96, P=0.0083$

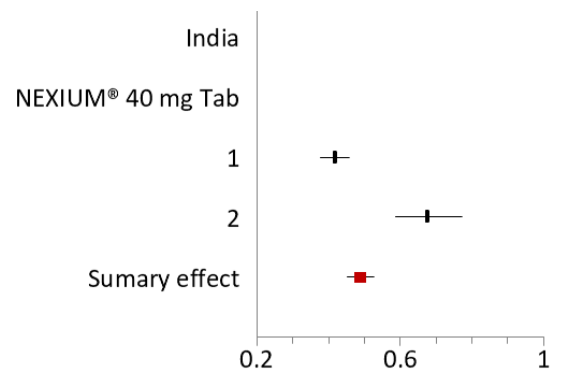


Foot note to Table 5: The vertical straight lines denote the GMRs of $\mathrm{AUC}_{0-\mathrm{t}}$ (fed/fasted) for each study and the lines on either side the $90 \%$ confidence intervals. The summary effect is represented by a red square and the lines on either side standing for point estimate and $95 \%$ confidence intervals, respectively, on the bottom line of each population. The $P$-value in summary statistics of each population corresponds to the variance of studies within that subgroup population and demonstrates whether the variance within subgroup is statistically significant. The $P$-value corresponding to $\mathrm{Q}_{\mathrm{Between}}$ on the bottom line of forest plot for each drug demonstrates whether the summary effect is the same for studies in North America as for the studies in India. PE, Point Estimate; CI, Confidence interval; $\tau^{2}$, measure of heterogeneity (between-studies variance); $I^{2}$, measure of heterogeneity (degree of inconsistency in $\%$ ).

$* 95 \%$ CI for summary food effect of each population.

Table 6. Summary statistics of omeprazole food effect using all available ANDS studies with Prilosec ${ }^{\circledR}$ $20 \mathrm{mg}$ capsule, Losec ${ }^{\circ} 20 \mathrm{mg}$ tablet and capsule

\section{AUC $_{0-\mathrm{t}}$ \\ PE (Fed/Fasted) \\ 90\% CI* \\ Food Effect}

North America

Losec ${ }^{2} 20 \mathrm{mg}$ Cap (2 x $\left.20 \mathrm{mg}\right)$

1

0.82

Losec ${ }^{2} 20 \mathrm{mg}$ Cap (1 x $\left.20 \mathrm{mg}\right)$

2

0.58

0.61

3

Summary effect $[95 \% \mathrm{CI}]$

0.64

Summary statistics: $P=0.256, \tau^{2}=0.008, I^{2}=26.62 \%$

\section{$\underline{\text { India }}$}

Losec ${ }^{\circledR} 20 \mathrm{mg}$ Tab $(1 \times 20 \mathrm{mg})$
1

2

3

4

Losec ${ }^{\circledR} 20 \mathrm{mg}$ Cap (1 x $\left.20 \mathrm{mg}\right)$

5

6
1.02

1.13

1.84

0.90

1.42

0.66
$[0.48,0.77]$
$[\mathbf{0 . 5 4}, \mathbf{0 . 7 6}]$

$[0.48,0.77]$
$[\mathbf{0 . 5 4}, \mathbf{0 . 7 6}]$

$[0.61,1.10]$

$[0.46,0.73]$

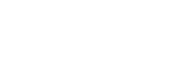

$[0.67,1,21]$

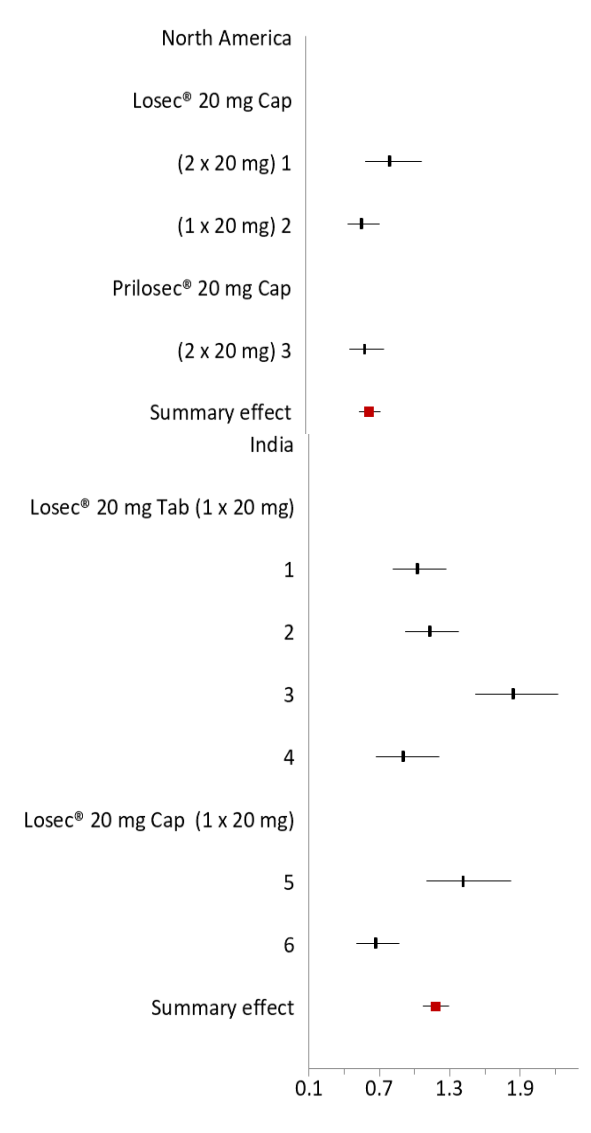

\section{Summary effect $[95 \% \mathrm{CI}] \quad \mathbf{1 . 1 8} \quad[1.06,1.32]$ \\ $[1.06,1.32]$}

$[1.10,1.82]$

$[0.51,0.87]$

Summary statistics: $P<0.0001, \tau^{2}=0.108, I^{2}=84.86$

Q-test for subgroup differences: $\mathrm{Q}_{\text {Between }}=34.0, P<0.0001$

Table 6 continues .... 


\section{$\mathrm{C}_{\max }$ PE (Fed/Fasted) \\ 90\% CI* \\ North America}

\section{Food Effect}

Losec $® 20$ mg Cap (2 x 20

$\mathrm{mg}$ )
1
0.65

$[0.53,0.81]$

Losec ${ }^{2} 20 \mathrm{mg}$ Cap (1 x $\left.20 \mathrm{mg}\right)$

20.33

$[0.28,0.38]$

Prilosec $® 20$ mg Cap (2 x 20 mg)

$3 \quad 0.53$

$[0.45,0.63]$

Summary effect [95\% CI]

0.45

$[0.40,0.51]$

Summary statistics: $P<0.0001, \tau^{2}=0.116, I^{2}=91.39 \%$

\section{India}

Losec ${ }^{\circledR} 20 \mathrm{mg}$ Tab (1 x $\left.20 \mathrm{mg}\right)$

$\begin{array}{ll}1 & 1.32 \\ 2 & 1.10 \\ 3 & 1.74 \\ 4 & 0.97\end{array}$

Losec ${ }^{2} 20 \mathrm{mg}$ Cap (1 x $\left.20 \mathrm{mg}\right)$

$\begin{array}{ll}5 & 0.84 \\ 6 & 0.49\end{array}$

\section{Summary effect [95\% CI]}

1.11

$[1.03,1.19]$

Summary statistics: $P<0.0001, \tau^{2}=0.191, I^{2}=95.96 \%$

Q-test for subgroup differences: $\mathrm{Q}_{\text {Between }}=165.76, P<0.0001$

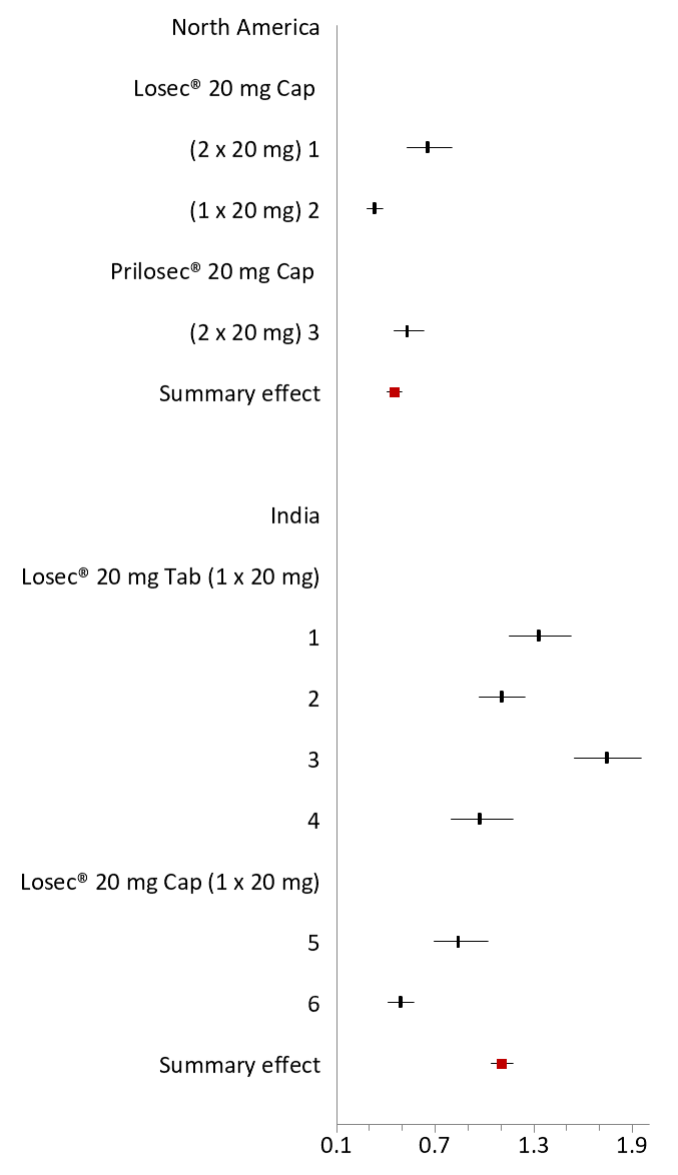

The vertical straight lines denote the GMRs of $\mathrm{AUC}_{0-\mathrm{t}}(\mathrm{fed} /$ fasted) for each study and the lines on either side the $90 \%$ confidence intervals. The summary effect is represented by a red square and the lines on either side standing for point estimate and $95 \%$ confidence intervals, respectively, on the bottom line of each population. The $P$-value in summary statistics of each population corresponds to the variance of studies within that subgroup population and demonstrates whether the variance within subgroup is statistically significant. The $P$-value corresponding to $\mathrm{Q}_{\text {Between }}$ on the bottom line of forest plot for each drug demonstrates whether the summary effect is the same for studies in North America as for the studies in India. PE, Point Estimate; CI, Confidence interval; $\tau^{2}$, measure of heterogeneity (between-studies variance); $I^{2}$, measure of heterogeneity (degree of inconsistency in \%).

$* 95 \%$ CI for summary food effect of each population. 
Table 7. Overall comparison (statistical and clinical) of calculated and labeled food effects for the studied products

\begin{tabular}{|c|c|c|c|c|c|c|c|c|c|c|}
\hline \multirow{2}{*}{ API } & \multirow[b]{2}{*}{$\begin{array}{c}\text { Drug } \\
\text { Product }\end{array}$} & \multirow[b]{2}{*}{$\begin{array}{c}\text { PK } \\
\text { Metric }\end{array}$} & \multirow[b]{2}{*}{$\mathbf{n}_{\mathbf{N A}}$} & \multirow[b]{2}{*}{ nIndia } & \multirow[b]{2}{*}{ Label } & \multirow[b]{2}{*}{$\begin{array}{c}\text { FE in North } \\
\text { America } \\
\text { Magnitude }^{\mathrm{a}}\end{array}$} & \multirow[b]{2}{*}{$\begin{array}{l}\text { FE in India } \\
\text { Magnitude }^{\mathrm{a}}\end{array}$} & \multicolumn{3}{|c|}{ 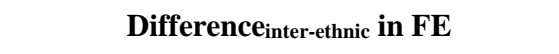 } \\
\hline & & & & & & & & $\begin{array}{c}\text { Difference } \\
\%^{\mathrm{b}}\end{array}$ & $\begin{array}{c}\text { Statistical } \\
\text { significance }^{c}\end{array}$ & $\begin{array}{c}\text { Possible } \\
\text { Clinical } \\
\text { relevance? }\end{array}$ \\
\hline \multirow{2}{*}{ Amiodarone } & \multirow{2}{*}{$\begin{array}{l}\text { Cordarone }{ }^{\circledR} \\
200 \mathrm{mg} \text { Tab }\end{array}$} & $\mathrm{AUC}_{0-\mathrm{t}}$ & \multirow{2}{*}{4} & \multirow{2}{*}{1} & \multirow{2}{*}{$\uparrow$} & $+116 \%$ & $+76 \%$ & $40 \%$ & \multirow{2}{*}{ Yes } & \multirow{2}{*}{ Yes } \\
\hline & & $\mathrm{C}_{\max }$ & & & & $+220 \%$ & $+148 \%$ & $72 \%$ & & \\
\hline \multirow{2}{*}{ Carbamazepine } & \multirow{2}{*}{$\begin{array}{c}\text { Tegretol }{ }^{\circledR} \text { CR } \\
400 \text { mg Tab }\end{array}$} & $\mathrm{AUC}_{0-\mathrm{t}}$ & \multirow{2}{*}{2} & \multirow{2}{*}{1} & \multirow{2}{*}{ No FE } & $+27 \%$ & $+15 \%$ & $12 \%$ & \multirow{2}{*}{ Yes } & \multirow{2}{*}{ No } \\
\hline & & $\mathrm{C}_{\max }$ & & & & $+29 \%$ & $+12 \%$ & $17 \%$ & & \\
\hline \multirow{2}{*}{ Diltiazem } & \multirow{2}{*}{$\begin{array}{l}\text { Tiazac® ER } \\
360 \text { mg Cap }\end{array}$} & $\mathrm{AUC}_{0-\mathrm{t}}$ & \multirow{2}{*}{1} & \multirow{2}{*}{2} & \multirow{2}{*}{ No FE } & - & $-2 \%$ & - & No & \multirow{2}{*}{ No } \\
\hline & & $\mathrm{C}_{\max }$ & & & & - & $+38 \%$ & - & - & \\
\hline \multirow{2}{*}{ Verapamil } & \multirow{2}{*}{$\begin{array}{l}\text { Isoptin® SR } \\
240 \mathrm{mg} \text { Tab }\end{array}$} & $\mathrm{AUC}_{0-\mathrm{t}}$ & \multirow{2}{*}{2} & 1 & & $+1 \%$ & $-28 \%$ & $29 \%$ & Yes & No \\
\hline & & $\mathrm{C}_{\max }$ & & 1 & $\downarrow$ & $-28 \%$ & $-50 \%$ & $22 \%$ & Yes & No \\
\hline Fsomenrazole & NEXIUM® & $\mathrm{AUC}_{0-\mathrm{t}}$ & 3 & 2 & 1 & $-46 \%$ & $-34 \%$ & $12 \%$ & Yes & No \\
\hline Esomeprazole & PR 40 mg Tab & $\mathrm{C}_{\max }$ & 3 & 2 & $\downarrow$ & $-60 \%$ & $-51 \%$ & $9 \%$ & res & NO \\
\hline Omenrazole & Losec $® 20$ & $\mathrm{AUC}_{0-\mathrm{t}}$ & 2 & 2 & $\mathrm{NoFF}$ & $-33 \%$ & $0 \%$ & $33 \%$ & Yes & No \\
\hline 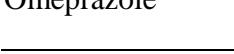 & mg Cap & $\mathrm{C}_{\max }$ & 2 & 2 & NOTE & $-58 \%$ & $-39 \%$ & $20 \%$ & Ies & No \\
\hline Omonronole & Losec $® 20$ & $\mathrm{AUC}_{0-\mathrm{t}}$ & & 4 & No $5 E_{-}$ & - & $+25 \%$ & - & & \\
\hline Silieplazvie & mg Tab & $\mathrm{C}_{\max }$ & - & 4 & NOTL & - & $+33 \%$ & - & - & - \\
\hline I anconrazole & Prevacid® 30 & $\mathrm{AUC}_{0-\mathrm{t}}$ & 1 & 4 & 1 & $-79 \%$ & $-71 \%$ & $8 \%$ & Yec & No \\
\hline Lansoprazore & mg Cap & $\mathrm{C}_{\max }$ & 1 & 4 & $\downarrow$ & $-84 \%$ & $-74 \%$ & $10 \%$ & res & NO \\
\hline Pantonrazole Na & Pantoloc®40 & $\mathrm{AUC}_{0-\mathrm{t}}$ & 2 & 6 & $\mathrm{NoFF}$ & $-16 \%$ & $-21 \%$ & $4 \%$ & No & No \\
\hline r alltopiazoit iva & mg Tab & $\mathrm{C}_{\max }$ & 2 & 0 & NOT & $-30 \%$ & $-19 \%$ & $11 \%$ & Yes & 1 No \\
\hline Pantonrazole Mo & Tecta® $40 \mathrm{mg}$ & $\mathrm{AUC}_{0-\mathrm{t}}$ & 1 & 1 & $\mathrm{NoFF}$ & $-23 \%$ & $+17 \%$ & $40 \%$ & Yes & Yes \\
\hline T antuptazoviting & Tab & $\mathrm{C}_{\max }$ & 1 & 1 & $1001 \mathrm{~L}$ & $-11 \%$ & $+10 \%$ & $21 \%$ & ICs & No \\
\hline Bahenrazole & 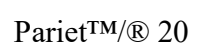 & $\mathrm{AUC}_{0-\mathrm{t}}$ & 2 & 3 & $\mathrm{NoFF}$ & $-20 \%$ & $+19 \%$ & $39 \%$ & Yes & No \\
\hline Nave piazoite & mg Tab & $\mathrm{C}_{\max }$ & 2 & $J$ & $1001 \mathrm{~L}$ & $-37 \%$ & $+47 \%$ & $84 \%$ & $1 \mathrm{Cs}$ & Yes \\
\hline
\end{tabular}




\begin{tabular}{|c|c|c|c|c|c|c|c|c|c|}
\hline \multirow{3}{*}{ API } & \multirow{3}{*}{$\begin{array}{l}\text { Drug } \\
\text { Product }\end{array}$} & \multirow{3}{*}{$\begin{array}{c}\text { PK } \\
\text { Metric }\end{array}$} & \multicolumn{2}{|c|}{ Fixed-Effect Model } & \multicolumn{2}{|c|}{ Random-Effects Model } & \multirow{2}{*}{\multicolumn{3}{|c|}{$\begin{array}{l}\text { Difference }_{\text {inter-ethnic }} \text { in FE } \\
\text { (Random-Effects Model) }\end{array}$}} \\
\hline & & & \multirow[t]{2}{*}{$\begin{array}{c}\text { FE in } \\
\text { North America } \\
\text { Magnitude }^{\mathrm{a}} \\
\end{array}$} & \multirow[t]{2}{*}{$\begin{array}{l}\text { FE in India } \\
\text { Magnitude }^{\text {a }}\end{array}$} & \multirow[t]{2}{*}{$\begin{array}{c}\text { FE in } \\
\text { North America } \\
\text { Magnitude }^{\mathrm{a}} \\
\end{array}$} & \multirow[t]{2}{*}{$\begin{array}{l}\text { FE in India } \\
\text { Magnitude a }^{*}\end{array}$} & & & \\
\hline & & & & & & & $\begin{array}{c}\text { Difference in \% } \\
\text { (Random-Effects } \\
\text { Model) }{ }^{\mathbf{b}}\end{array}$ & $\begin{array}{c}\text { Statistical } \\
\text { significance }^{c}\end{array}$ & $\begin{array}{l}\text { Possible Clinical } \\
\text { relevance? }{ }^{\mathrm{d}}\end{array}$ \\
\hline \multirow{2}{*}{ Amiodarone } & \multirow{2}{*}{$\begin{array}{l}\text { Cordaron }{ }^{\circledR} \\
200 \mathrm{mg} \text { Tab }\end{array}$} & $\mathrm{AUC}_{0-\mathrm{t}}$ & $+116 \%$ & $+76 \%$ & $+118 \%$ & $+76 \%$ & $42 \%$ & \multirow{2}{*}{ Yes } & \multirow{2}{*}{ Yes } \\
\hline & & $\mathrm{C}_{\max }$ & $+220 \%$ & $+148 \%$ & $+220 \%$ & $+148 \%$ & $72 \%$ & & \\
\hline \multirow{2}{*}{ Carbamazepine } & \multirow{2}{*}{$\begin{array}{c}\text { Tegretol }{ }^{\circledR} \\
\text { CR } 400 \mathrm{mg} \\
\text { Tab }\end{array}$} & $\mathrm{AUC}_{0-\mathrm{t}}$ & $+27 \%$ & $+15 \%$ & $+31 \%$ & $+15 \%$ & $16 \%$ & \multirow{2}{*}{ No* } & \multirow{2}{*}{ No } \\
\hline & & $\mathrm{C}_{\max }$ & $+29 \%$ & $+12 \%$ & $+33 \%$ & $+12 \%$ & $21 \%$ & & \\
\hline \multirow{2}{*}{ Diltiazem } & \multirow{2}{*}{$\begin{array}{l}\text { Tiazac }{ }^{\circ} \\
\text { ER } 360 \mathrm{mg} \\
\quad \text { Cap }\end{array}$} & $\mathrm{AUC}_{0-\mathrm{t}}$ & - & $-2 \%$ & - & $-2 \%$ & - & No & \multirow{2}{*}{ No } \\
\hline & & $\mathrm{C}_{\max }$ & - & $+38 \%$ & - & $+36 \%$ & - & - & \\
\hline \multirow{2}{*}{ Verapamil } & \multirow{2}{*}{$\begin{array}{c}\text { Isoptin }{ }^{\circledR} \\
\text { SR } 240 \mathrm{mg} \\
\text { Tab }\end{array}$} & $\mathrm{AUC}_{0-\mathrm{t}}$ & $+1 \%$ & $-28 \%$ & $+1 \%$ & $-29 \%$ & $29 \%$ & \multirow{2}{*}{ Yes } & \multirow{2}{*}{ No } \\
\hline & & $\mathrm{C}_{\max }$ & $-28 \%$ & $-50 \%$ & $-28 \%$ & $-50 \%$ & $21 \%$ & & \\
\hline \multirow{2}{*}{ Esomeprazole } & \multirow{2}{*}{$\begin{array}{l}\text { Nexium }{ }^{\circledR} \\
\text { PR } 40 \mathrm{mg} \\
\text { Tab }\end{array}$} & $\mathrm{AUC}_{0-\mathrm{t}}$ & $-46 \%$ & $-34 \%$ & $-48 \%$ & $-27 \%$ & $21 \%$ & \multirow{2}{*}{ No* } & \multirow{2}{*}{ No } \\
\hline & & $\mathrm{C}_{\max }$ & $-60 \%$ & $-51 \%$ & $-61 \%$ & $-47 \%$ & $14 \%$ & & \\
\hline \multirow{2}{*}{ Omeprazole } & \multirow{2}{*}{$\begin{array}{c}\text { Losec } ® 20 \\
\text { mg Cap }\end{array}$} & $\mathrm{AUC}_{0-\mathrm{t}}$ & $-33 \%$ & $0 \%$ & $-42 \%$ & $4 \%$ & $38 \%$ & \multirow{2}{*}{ Yes } & \multirow{2}{*}{ No } \\
\hline & & $\mathrm{C}_{\max }$ & $-58 \%$ & $-39 \%$ & $-67 \%$ & $-36 \%$ & $31 \%$ & & \\
\hline$J$ & Prevacid® & $\mathrm{AUC}_{0-\mathrm{t}}$ & $-79 \%$ & $-71 \%$ & $-79 \%$ & $-73 \%$ & $6 \%$ & $\mathrm{Y}$ & $\mathrm{N}$ \\
\hline Lansoprazore & 30 mg Cap & $\mathrm{C}_{\max }$ & $-84 \%$ & $-74 \%$ & $-84 \%$ & $-77 \%$ & $7 \%$ & res & NO \\
\hline Pantonrazole $\mathrm{Na}$ & Pantoloc $® 4$ & $\mathrm{AUC}_{0-\mathrm{t}}$ & $-16 \%$ & $-21 \%$ & $-16 \%$ & $-20 \%$ & $4 \%$ & No & No \\
\hline Pantoprazole Na & 0 mg Tab & $\mathrm{C}_{\max }$ & $-30 \%$ & $-19 \%$ & $-30 \%$ & $-19 \%$ & $11 \%$ & Yes & No \\
\hline Pantonrazole Ma & Tecta® 40 & $\mathrm{AUC}_{0-\mathrm{t}}$ & $-23 \%$ & $+17 \%$ & $-23 \%$ & $+17 \%$ & $40 \%$ & & Yes \\
\hline Fantoprazore ing & mg Tab & $\mathrm{C}_{\max }$ & $-11 \%$ & $+10 \%$ & $-11 \%$ & $+10 \%$ & $21 \%$ & res & No \\
\hline D 1 - -8 & Pariet & $\mathrm{AUC}_{0-\mathrm{t}}$ & $-20 \%$ & $+19 \%$ & $-9 \%$ & $+17 \%$ & $26 \%$ & & No \\
\hline Карергаzоте & $20 \mathrm{mg}$ Tab & $\mathrm{C}_{\max }$ & $-37 \%$ & $+47 \%$ & $-12 \%$ & $+31 \%$ & $43 \%$ & No & Yes \\
\hline
\end{tabular}

Footnotes to Table 7 and 8 . $\uparrow$ and $\downarrow$ arrows denote increased and decreased exposure with food, respectively; $\mathrm{n}_{\mathrm{NA}}$; Number of ANDSs in North American population; $\mathrm{n}_{\text {India, }}$

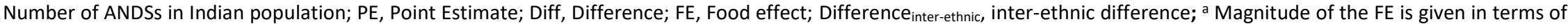
the percentage of the difference between fed and fasted (\% Difference $=\frac{\text { Fed }- \text { Fasted }}{\text { Fasted }} \times 100$ ); ${ }^{b}$ When food impacted exposure in the same direction in both populations, the difference in their $\mathrm{FE}$ was calculated as: \%Difference ${ }_{\text {inter-ethnic }}=\left|\% \mathrm{FE}_{\text {North American }}-\% \mathrm{FE}_{\text {Indian }}\right|$. When food impacted exposure in two different directions, the difference in $\mathrm{FE}$ between two populations was calculated by summing the absolute value of $\% \mathrm{FE}$ in each population: \%Difference inter-ethnic $=\left|\% \mathrm{FE}_{\text {North American }}\right|+\left|\% \mathrm{FE}_{\text {Indian }}\right| ;{ }^{\mathrm{c}} \mathrm{Statistically}$ significant difference in summary food effect between the two geographical/ethnic subgroups was concluded based on subgroup analysis ( $Q$-test, $P<0.05) ; *$ When there was a

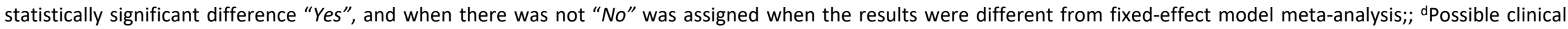
relevance was concluded when \%Difference inter-ethnic in FE $\geq 40 \%$. When there was a clinically significant difference "Yes", and when there was not "No" was assigned. 


\section{DISCUSSION}

This study aimed to investigate if two formulations assessed as being bioequivalent in one geographic/ethnic population would also be bioequivalent in another one. Food effects for the same reference product were calculated and compared between populations. At this point in time we could only find data available for two different populations, North American and Indian.

The most striking differences in FEs between the two populations were observed with rabeprazole and amiodarone. For rabeprazole, an increased exposure with food was observed in India, while a decrease was observed in North America. The difference in FEs between the two regions was found to be of both statistical $(P<0.05)$ and possible clinical significance (\% Diff inter-ethnic $\geq 40 \%)$. The observed $\mathrm{FE}$ on $\mathrm{C}_{\max }$ was more apparent in both populations with Point Estimates (PEs: fed/ fasted ratios) falling completely out of the $80-125 \%$ usual equivalence limits. High between-study variability was only observed for the Indian population rabeprazole studies $\left(I^{2}=\right.$ $90 \%$ for $\mathrm{AUC}_{0-\mathrm{t}}, 95 \%$ for $\mathrm{C}_{\max }$ ). Two of the three available rabeprazole submissions using Indian populations (\#2 and \#3) were in general agreement with each other, as significant increases in $\mathrm{C}_{\max }$ were observed with PEs falling above the usual $125 \%$ upper limit. Submission\#1 with no $\mathrm{FE}$ on $\mathrm{C}_{\max }$ was however in contradiction with the other two. In contrast, very low to negligible between-study variability in FEs were observed for theubmissions using the North American populations $(\mathrm{I} 2=20.9 \%$ for $\mathrm{Cmax}$ and $\mathrm{I} 2=0 \%$

for AUC0-t). As such, the significant differences observed in the FEs for rabeprazole between these two populations do not seem to be due to between-study variability. For amiodarone, increased exposure with food was observed in both populations; however, the difference in FEs between the two populations were still statistically significant $(\mathrm{P}<0.05)$ and of possible clinical relevance $(40 \%$ and $72 \%$ larger increase in North America than in India in terms of AUC0-t and Cmax, respectively).
Similarly to rabeprazole, a difference in the direction of the FE between the two populations was also observed for pantoprazolemagnesium. An increase in exposure in terms of $\mathrm{AUC}_{0-\mathrm{t}}$ with food was observed in Indian populations, while a decrease was observed in North American ones. This difference in $\mathrm{AUC}_{0-\mathrm{t}}$ was statistically significant $(P<0.05)$ and possibly clinically relevant.

To date, many interactions between pharmaceutical excipients and transporters/CYP enzymes have been documented. For the studied drugs several of the excipients are known for their inteaction with enzymes and transporters, and include Tween 80 (40), SLS (19, 21), and PEG $(18,41-44)$ in lansoprazole (PREVACID® Cap); magnesium-stearate $(45,46)$ in esomeprazole (NEXIUM $\left.{ }^{\circledR} \quad \mathrm{Tab}\right)$ and amiodarone (CORDARONE® Tab); and PEG in esomeprazole (NEXIUM ${ }^{\circledR}$ Tab) and omeprazole (LOSEC $®$ Cap). CYP3A4 inhibition by polysorbates (Tween $\AA$ ) has also been demonstrated $(40,47)$, with inhibition of human cDNA expressed CYP3A4 at concentrations of $0.005 \%$ and above (40). In an in vitro human colon and liver cell lines study, magnesiumstearate was shown to decrease CYP3A4 mRNA expression by more than $40 \%$ (45). Similarly, common pharmaceutical excipients have been shown to inhibit or at least attenuate P-gp function by more than fivefold (16). Cosolvents (e.g., PEG 400) (48), the Cremophor ${ }^{\circledR}$ class of pharmaceutical excipients (e.g., Cremophor EL) $(49,50)$, Tween ${ }^{\circledR} 20$, and Tween ${ }^{\circledR} 80$ have been identified as P-gp inhibitors $(49,51)$ and were found to enhance the transfer of P-gp substrate, digoxin, across the intestinal mucosa in different in vitro cell models by $\approx 2$ folds (51). In the meantime, lower level of expression and lower CYP2C19 (23-25, 55-56), CYP2D6 (57-59), CYP2C9 (22), and P-gp transporters (26) in Asians/Indians versus Caucasians/North Americans have been reported. The fourfold larger omeprazole AUC in Asian patients compared to Caucasian ones, indicated in the label (60), supports this information. Mannitol (61) in rabeprazole formulation (Pariet ${ }^{\circledR} \mathrm{Tab}$ ), and magnesium stearate (45-46) in 
(Cordarone ${ }^{\circledR} \mathrm{Tab}$ ) formulations are excipients known to influence drugbioavailability and bioequivalence. Rabeprazole has been reported to be metabolized by polymorphically expressed CYP2C19 and CYP3A4 enzymes. Similarly, amiodarone is a major substrate of CYP3A, but also of CYP2D6 and P-gp (62). The differences observed in the rabeprazole and amiodarone FEs between the North American and Indian populations may be hypothesized to be partly attributed to the different prevalence of the variants of CYP enzymes and P-gp transporters present in the two populations, and to the influence of excipients on bioavailability through the interaction with CYP enzymes and transporters, but this of course would need to be verified in further studies.

For five of the study drugs where food impacted exposure in the same direction in both populations, the effect of food on exposure in terms of both $\mathrm{AUC}_{0-\mathrm{t}}$ and $\mathrm{C}_{\max }$ in North American populations was numerically larger than in the Indian one. For instance, $33 \%$ and $20 \%$ larger decrease in omeprazole $\mathrm{AUC}_{0-\mathrm{t}}$ and $\mathrm{C}_{\max }$ with food, respectively, was observed in the North American versus Indian populations. Omeprazole, esomeprazole, and lansoprazole have been reported to be mainly metabolized by CYP2C19 and also by CYP3A4 enzymes; omeprazole and pantoprazole are reported to be also metabolized by CYP2D6 to a lesser extent. Although not widely investigated, some studies suggest that proton pump inhibitors (PPIs) are also substrates of P-gp transporters (63). The larger FE observed in North American populations versus the Indian ones could be hypothesized to be partly due to the reportedly lower levels of expression/ function of CYP enzymes/P-gp transporters in Indians, but this also would need to be verified in further studies.

The excipients mentioned above are only some of the examples that have been previously indicated to have an impact on the function of transporters and enzymes. Excipients can influence bioavailability, and therefore may impact bioequivalence outcomes via different mechanisms. Excipient's type, how they are combined, and their concentrations may influence the PK and physicochemical characteristics of APIs (19, 21, 64-65). For instance, drugs with high permeability are often assumed to be less susceptible to excipient influence on their bioavailability than drugs with low permeability, and therefore the influence of excipients on bioequivalence between their formulations is expected to be of no significance (66). Published data may contradict this assumption, however, because some show that the influence of excipients on bioequivalence outcomes can be unpredictable. A clinical study with remarkable importance in undermining this traditional assumption is the bioequivalence study of a highly permeable, highly soluble drug, risperidone's oral solution (19). In that study, a manufacturer developed two oral test solutions of risperidone containing 50 and $7 \mathrm{mg} / \mathrm{ml}$ of sorbitol in addition to the same qualitative and quantitative excipients included in the reference product (Risperdal ${ }^{\circledR} 1$ $\mathrm{mg} / \mathrm{ml}$ oral solution). Both Test solutions failed to show bioequivalence with the reference product, and this is despite the low intra-subject variability and sufficient study power. Commonly used excipients can therefore impact not only the bioavailability and bioequivalence outcomes for drugs with low permeability, but also those with high permeability and high solubility. Excipients can impact bioavailability and bioequivalence, even solutions, where the release of the drug substance from the drug product has been considered to be self-evident. There are several other examples demonstrating the unpredictable impact of excipients on bioequivalence outcomes (19, 20, 64, 67).

In the evaluation of heterogeneity, less than $11.1 \%$ and $44.4 \%$ of the studies conducted with the North American population were associated with significant inconsistency $\left(I^{2} \geq 75 \%\right)$ in $\mathrm{FE}$ results in terms of the $\mathrm{AUC}_{0-\mathrm{t}}$ and $\mathrm{C}_{\max }$, respectively, while $71.4 \%$ of the studies conducted with Indian populations had significant inconsistency for both exposure 
measures. Larger between-study variabilities $\left(I^{2}\right)$ for all PPIs were found in studies conducted in Indian populations (Table 4). Greater prevalence of polymorphism in CYP enzymes has been reported in Indian versus North American populations $(23-25,55,56)$. This may be a reason explaining the higher heterogeneity seen in Indian populations, but this also would need to be confirmed in further studies.

We concluded summary $\mathrm{FE}$ in each population by prioritizing fixed-effect, rather than random-effects model meta-analysis as fixed-effect model is used when all studies in the meta-analysis are drawn from a common population and all factors which could influence the effect size (here, the FE in each ANDS study) are the same in all the study populations. In our analysis, the FE in each study was calculated based on the data extracted from an ANDS. Therefore, all studies were expected to share the same study design in order to meet the regulatory requirements (e.g., the same RLD in the same ethnic population, the same volume of water for dose administration, the same meal with the same food volume and viscosity, etc.). As a result, all variables with a potential impact on effect size were assumed to be the same across the studies. This complies with the principles of fixed-effect model.

Due to the large observed between-study variability in some studies, especially in those conducted in Indian populations, one may question whether the observed inter-ethnic differences in FEs were the true differences in PEs (fed/fasted) or they could simply be confounded by between-study variability. In order to account for between-study variability, we also conducted a random-effect model metaanalysis to calculate the summary FEs for each study drug product (data not shown). The results were in general agreement with the fixed-effect model meta-analysis. The same inter-ethnic differences were concluded for all study drugs from a clinical significance standpoint. It suggested that the observed inter-ethnic differences in FEs are unlikely to be due to between-study variability. A comparison of summary FEs calculated from fixed-effect and random-effect model meta-analyses for each drug in each population is provided in Table 8.
The examples in this study suggest that possible differences in FEs between geographical/ethnic populations may exist for certain drugs, regardless of the underlying mechanisms. Although unlikely because their specific caloric breakdown to protein, carbohydrate, and fat contents are the same, some may attribute these inter-ethnic differences in FEs to the different meal contents between the North American versus Indian studies. When conducted in India or North America, bioequivalence studies for the purpose of generic submission to the North American regulatory agencies (i.e., the FDA and Health Canada (HC)) must comply with the standards set in terms of meal contents by the US FDA and HC. Minor differences in the test meal are expected to have no impact on FE and are accepted by regulatory agencies. As per the FDA Guidance (10) substitutions in the test meal can be made as long as the meal provides a similar amount of calories from protein, carbohydrate, and fat and has comparable meal volume and viscosity. In summary, the observed differences in FEs between the two populations should not be due to a difference in meal contents between the studies conducted in North America and India. And even if they were, how unlikely it may be, the end result would be the same in terms of not being able to extrapolate $\mathrm{BE}$ results from one population to the next.

Our study suggested possible differences in FE between two populations for nine drug products. If these results can be confirmed by others, then other differences are likely to be found among the thousands of other marketed drug products. The FEs observed for the reference products using North American populations were in general different than those using Indian populations, implying that two formulations that are assessed as bioequivalent in one population may not necessarily be bioequivalent in another one. This is in contradiction with the traditional view that bioequivalence outcomes should not differ between populations when a study is using a crossover design. 
Central to the conduct of bioequivalence studies in HVs, is the extrapolation of bioequivalence outcomes from healthy populations to patients. It is based on the assumption that (1) the use of HVs will minimize both inter- and intra-subject variability, and that (2) the equivalence observed between two products under healthy conditions can be extrapolated to the disease conditions. This may not always be appropriate. To date, different studies in human subjects and animal models have revealed that bioequivalence or PK equivalence observed under healthy conditions may not always translate to equivalence under experimentally altered conditions or disease conditions (2-8, 68-71). For example, levothyroxine is a drug whose bioavailability is affected by altered gastrointestinal conditions, which might be present in a patient population. Several studies have documented that the elevated gastric $\mathrm{pH}$, either due to impaired gastric acid secretion (71) or gastric $\mathrm{pH}$ altering drugs, such as PPIs $(3,4,6,68)$, reduced the oral bioavailability of levothyroxine significantly. In a levothyroxine PK study (two-way crossover) conducted in human subjects $(n=15)$ (6) levothyroxine capsules (Tirosint ${ }^{\circledR}$ capsule) and levothyroxine tablets (Synthroid® tablet) assessed as being PK equivalent under fasted conditions prior to the intravenous administration of esomeprazole, were found to not be PK

\section{REFERENCES}

1. Jamali F, Aghazadeh-Habashi A. Rapidly dissolving formulations for quick absorption during pain episodes: ibuprofen. International journal of clinical pharmacology and therapeutics. 2008;46(2):55-63.

2. Jamali F, Kunz-Dober CM. Pain-mediated altered absorption and metabolism of ibuprofen: an explanation for decreased serum enantiomer concentration after dental surgery. British journal of clinical pharmacology. 1999;47(4):391-6.

3. Pabla D, Akhlaghi F, Zia H. A comparative pH-dissolution profile study of selected commercial levothyroxine products using inductively coupled plasma mass spectrometry. European journal of pharmaceutics and biopharmaceutics : official journal of Arbeitsgemeinschaft fur equivalent under altered gastric $\mathrm{pH}$ conditions caused by prior intravenous administration of esomeprazole. The PK studies (two-way crossover) of omeprazole in 40 (7) and 23 (8) human subjects also demonstrated that differences between two formulations may remain hidden or nonsignificant under one condition, while they may be accentuated under another.

In conclusion, we suggest that extrapolating bioequivalence study results from one population/region to another may not always be appropriate. We acknowledge that the detected discrepancies in our calculated FEs between the two populations may not always be clinically relevant. Nevertheless, we also found inter-ethnic differences in FE, which may be of clinical significance for drugs with fatal side-effects such as amiodarone (62) and verapamil (72). Further studies and research in this field should be undertaken.

\section{CONFLICT OF INTEREST}

This study was supported by Learn and Confirm Inc. and was part of the PhD research work of Deniz Ozdin under the direction of M Ducharme, $F$ Varin and A Fuglsang. The authors declare that they have no conflict of interest related to this work.

Pharmazeutische Verfahrenstechnik eV. 2009;72(1):105-10.

4. Sachmechi I, Reich DM, Aninyei M, Wibowo F, Gupta G, Kim PJ. Effect of proton pump inhibitors on serum thyroid-stimulating hormone level in euthyroid patients treated with levothyroxine for hypothyroidism. Endocrine practice : official journal of the American College of Endocrinology and the American Association of Clinical Endocrinologists. 2007;13(4):345-9.

5. Aghazadeh-Habashi A, Jamali F. Pharmacokinetics of meloxicam administered as regular and fast dissolving formulations to the rat: influence of gastrointestinal dysfunction on the relative bioavailability of two formulations. European journal of pharmaceutics and biopharmaceutics : official journal of Arbeitsgemeinschaft fur 
Pharmazeutische Verfahrenstechnik eV. 2008;70(3):889-94.

6. Seng Yue C, Benvenga S, Scarsi C, Loprete L, Ducharme MP. When Bioequivalence in Healthy Volunteers May not Translate to Bioequivalence in Patients: Differential Effects of Increased Gastric $\mathrm{pH}$ on the Pharmacokinetics of Levothyroxine Capsules and Tablets. Journal of pharmacy \& pharmaceutical sciences : a publication of the Canadian Society for Pharmaceutical Sciences, Societe canadienne des sciences pharmaceutiques. 2015;18(5):844-55.

7. Elkoshi Z, Behr D, Mirimsky A, Tsvetkov I, Danon A. Multiple-Dose Studies can be a More Sensitive Assessment for Bioequivalence than Single-Dose Studies : The Case with Omeprazole. Clinical drug investigation. 2002;22(9):585-92.

8. Vaz-da-Silva M, Loureiro AI, Nunes T, Maia J, Tavares S, Falcao A, et al. Bioavailability and bioequivalence of two enteric-coated formulations of omeprazole in fasting and fed conditions. Clinical drug investigation. 2005;25(6):391-9.

9. Health Canada, Guidance Document Comparative Bioavailability Standards: Formulations Used for Systemic Effects, 2018. Last Accessed 5 April 2019, at https://www.canada.ca/content/dam/hcsc/migration/hc-sc/dhpmps/alt_formats/pdf/prodpharma/applicdemande/guide-ld/bio/comparativebioavailability-standards-formulations-usedsystemic-effects.pdf

10.US-FDA, CDER: Guidance for Industry, Bioequivalence Studies with Pharmacokinetic Endpoints for Drugs Submitted Under an ANDA, 2013. Last accessed 7 February 2019, at:

https://www.fda.gov/media/87219/download.

11.EMA Guideline on the Investigation of Bioequivalence. August 2010. Last accessed 6 March 2019 at https://www.ema.europa.eu/en/documents/sci entific-guideline/guideline-investigationbioequivalence-rev1_en.pdf.

12. Hilal-Dandan R, Brunton LL. Membrane Transporters and Drug Response. Goodman and Gilman's Manual of Pharmacology and
Therapeutics, 2e. New York, NY: McGrawHill Education; 2016.

13. Scherrmann JM. Transporters in absorption, distribution, and elimination. Chemistry \& biodiversity. 2009;6(11):1933-42.

14. Thiebaut F, Tsuruo T, Hamada H, Gottesman MM, Pastan I, Willingham MC. Cellular localization of the multidrug-resistance gene product P-glycoprotein in normal human tissues. Proceedings of the National Academy of Sciences of the United States of America. 1987;84(21):7735-8.

15.Kanfer I, Shargel L. IntroductionBioequivalence Issues. In: Kanfer I, Shargel L, editors. Generic drug product development : bioequivalence issues. New York: Informa Healthcare; 2008. p. 1-14.

16.Zhang W, Li Y, Zou P, Wu M, Zhang Z, Zhang T. The Effects of Pharmaceutical Excipients on Gastrointestinal Tract Metabolic Enzymes and Transporters-an Update. The AAPS Journal. 2016;18(4):83043.

17.Goole J, Lindley DJ, Roth W, Carl SM, Amighi K, Kauffmann JM, et al. The effects of excipients on transporter mediated absorption. International journal of pharmaceutics. 2010;393(1-2):17-31.

18. Buggins TR, Dickinson PA, Taylor G. The effects of pharmaceutical excipients on drug disposition. Advanced drug delivery reviews. 2007;59(15):1482-503.

19. Garcia-Arieta A. Interactions between active pharmaceutical ingredients and excipients affecting bioavailability: impact on bioequivalence. European journal of pharmaceutical sciences : official journal of the European Federation for Pharmaceutical Sciences. 2014;65:89-97.

20.Chen ML, Straughn AB, Sadrieh N, Meyer M, Faustino PJ, Ciavarella AB, et al. A modern view of excipient effects on bioequivalence: case study of sorbitol. Pharmaceutical research. 2007;24(1):73-80.

21.Vaithianathan S, Haidar SH, Zhang X, Jiang W, Avon C, Dowling TC, et al. Effect of Common Excipients on the Oral Drug Absorption of Biopharmaceutics Classification System Class 3 Drugs Cimetidine and Acyclovir. Journal of 
pharmaceutical sciences. 2016;105(2):9961005.

22.Limdi NA, McGwin G, Goldstein JA, Beasley TM, Arnett DK, Adler BK, et al. Influence of CYP2C9 and VKORC1 1173C/T genotype on the risk of hemorrhagic complications in African-American and European-American patients on warfarin. Clinical pharmacology and therapeutics. 2008;83(2):312-21.

23. Yasuda S, Zhang L, Huang S-M. The Role of Ethnicity in Variability in Response to Drugs: Focus on Clinical Pharmacology Studies. Clinical Pharmacology \& Therapeutics. 2008;84(3):417-23.

24.Sohn DR, Kobayashi K, Chiba K, Lee KH, Shin SG, Ishizaki T. Disposition kinetics and metabolism of omeprazole in extensive and poor metabolizers of S-mephenytoin 4'hydroxylation recruited from an Oriental population. The Journal of pharmacology and experimental therapeutics. 1992;262(3):1195202.

25.Chang M, Tybring G, Dahl ML, Gotharson E, Sagar M, Seensalu R, et al. Interphenotype differences in disposition and effect on gastrin levels of omeprazole--suitability of omeprazole as a probe for CYP2C19. British journal of clinical pharmacology. 1995;39(5):511-8.

26.Balram C, Sharma A, Sivathasan C, Lee EJD. Frequency of C3435T single nucleotide MDR1 genetic polymorphism in an Asian population: phenotypic-genotypic correlates. British journal of clinical pharmacology. 2003;56(1):78-83.

27.Kenyon CJ, Brown F, McClelland GR, Wilding IR. The use of pharmacoscintigraphy to elucidate food effects observed with a novel protease inhibitor (saquinavir). Pharmaceutical research. 1998;15(3):417-22.

28. Ducharme MP, Warbasse LH, Edwards DJ. Disposition of intravenous and oral cyclosporine after administration with grapefruit juice. Clinical pharmacology and therapeutics. 1995;57(5):485-91.

29.Gupta SK, Manfro RC, Tomlanovich SJ, Gambertoglio JG, Garovoy MR, Benet LZ. Effect of food on the pharmacokinetics of cyclosporine in healthy subjects following oral and intravenous administration. Journal of clinical pharmacology. 1990;30(7):643-53.
30.Gupta SK, Benet LZ. High-fat meals increase the clearance of cyclosporine. Pharmaceutical research. 1990;7(1):46-8.

31. Health Canada. Drug Product database online query. Last accessed July 62020 at: https://health-products.canada.ca/dpdbdpp/index-eng.jsp.

32.US FDA Drug Product Database for FDA Approved Drug Products. 2018 Available from:

https://www.accessdata.fda.gov/scripts/cder/d af/.

33.Fuglsang A. Sequential bioequivalence approaches for parallel designs. The AAPS journal. 2014;16(3):373-8.

34. Schuirmann DJ. A comparison of the Two One-Sided Tests Procedure and the Power Approach for assessing the equivalence of average bioavailability. Journal of Pharmacokinetics and Biopharmaceutics. 1987;15(6):657-80.

35.Michael Borenstein, Larry V. Hedges, Julian P. T. Higgins, Rothstein HR. Introduction to Meta-Analysis: John Wiley \& Sons, Ltd.; 2009.

36. Handbook of Drug-Nutrient Interactions. 2 ed. J.I. Boullata, Armenti VT, editors: Humana Press; 2010.

37.FDA, Drug Development and Drug Interactions: Table of Substrates, Inhibitors and Inducers. Last accessed 28 June 2019 at: https://www.fda.gov/drugs/drug-interactionslabeling/drug-development-and-druginteractions-table-substrates-inhibitors-andinducers.

38. Krishna R, Yu L. Biopharmaceutics Applications in Drug Development. New York: Springer; 2008.

39. You G, Morris ME. Drug Transporters: Molecular characterization and role in drug disposition. Hoboken: Wyley; 2014.

40.Mountfield RJ, Senepin S, Schleimer M, Walter I, Bittner B. Potential inhibitory effects of formulation ingredients on intestinal cytochrome $\mathrm{P} 450$. International journal of pharmaceutics. 2000;211(1-2):89-92.

41.Basit AW, Podczeck F, Newton JM, Waddington WA, Ell PJ, Lacey LF. Influence of polyethylene glycol 400 on the gastrointestinal absorption of ranitidine. 
Pharmaceutical research. 2002;19(9):136874.

42.Ashiru DA, Patel R, Basit AW. Polyethylene glycol 400 enhances the bioavailability of a BCS class III drug (ranitidine) in male subjects but not females. Pharmaceutical research. 2008;25(10):2327-33.

43.Singh P, Guillory JK, Sokoloski TD, Benet LZ, Bhatia VN. Effect of inert tablet ingredients on drug absorption. I. Effect of polyethylene glycol 4000 on the intestinal absorption of four barbiturates. Journal of pharmaceutical sciences. 1966;55(1):63-8.

44.Ren X, Mao X, Si L, Cao L, Xiong H, Qiu J, et al. Pharmaceutical excipients inhibit cytochrome $\mathrm{P} 450$ activity in cell free systems and after systemic administration. European journal of pharmaceutics and biopharmaceutics : official journal of Arbeitsgemeinschaft fur Pharmazeutische Verfahrenstechnik eV. 2008;70(1):279-88.

45.Tompkins L, Lynch C, Haidar S, Polli J, Wang H. Effects of Commonly Used Excipients on the Expression of CYP3A4 in Colon and Liver Cells. Pharmaceutical research. 2010;27(8):1703-12.

46.Richards RM, Xing JZ, Mackay KM. Excipient interaction with cetylpyridinium chloride activity in tablet based lozenges. Pharmaceutical research. 1996;13(8):125864.

47.Bravo Gonzalez RC, Huwyler J, Boess F, Walter I, Bittner B. In vitro investigation on the impact of the surface-active excipients Cremophor EL, Tween 80 and Solutol HS 15 on the metabolism of midazolam. Biopharmaceutics \& drug disposition. 2004;25(1):37-49.

48. Ashiru-Oredope DA, Patel N, Forbes B, Patel $\mathrm{R}$, Basit AW. The effect of polyoxyethylene polymers on the transport of ranitidine in Caco-2 cell monolayers. International journal of pharmaceutics. 2011;409(1-2):164-8.

49.Hanke U, May K, Rozehnal V, Nagel S, Siegmund W, Weitschies W. Commonly used nonionic surfactants interact differently with the human efflux transporters ABCB1 (pglycoprotein) and ABCC2 (MRP2). European journal of pharmaceutics and biopharmaceutics : official journal of
Arbeitsgemeinschaft fur Pharmazeutische Verfahrenstechnik eV. 2010;76(2):260-8.

50.Zhao G, Huang J, Xue K, Si L, Li G. Enhanced intestinal absorption of etoposide by selfmicroemulsifying drug delivery systems: roles of P-glycoprotein and cytochrome P450 3A inhibition. European journal of pharmaceutical sciences : official journal of the European Federation for Pharmaceutical Sciences. 2013;50(3-4):429-39.

51.Cornaire G, Woodley J, Hermann P, Cloarec A, Arellano C, Houin G. Impact of excipients on the absorption of P-glycoprotein substrates in vitro and in vivo. International journal of pharmaceutics. 2004;278(1):119-31.

52.Xie HG, Kim RB, Wood AJ, Stein CM. Molecular basis of ethnic differences in drug disposition and response. Annual review of pharmacology and toxicology. 2001;41:81550.

53.Kim K, Johnson JA, Derendorf H. Differences in drug pharmacokinetics between East Asians and Caucasians and the role of genetic polymorphisms. Journal of clinical pharmacology. 2004;44(10):1083-105.

54.Simeprevir Clinical Pharmacology and Biopharmaceutics Review. NDA 205-123. Last accessed 16 December 2018, at https://www.accessdata.fda.gov/drugsatfda_d ocs/nda/2013/205123Orig1s000ClinPharmR. pdf.

55.Benet LZ. The drug transporter-metabolism alliance: uncovering and defining the interplay. Molecular pharmaceutics. 2009;6(6):1631-43.

56.de Morais SM, Wilkinson GR, Blaisdell J, Nakamura K, Meyer UA, Goldstein JA. The major genetic defect responsible for the polymorphism of S-mephenytoin metabolism in humans. The Journal of biological chemistry. 1994;269(22):15419-22.

57.Relling MV, Cherrie J, Schell MJ, Petros WP, Meyer WH, Evans WE. Lower prevalence of the debrisoquin oxidative poor metabolizer phenotype in American black versus white subjects. Clinical Pharmacology \& Therapeutics. 1991;50(3):308-13.

58.Evans WE, Relling MV, Rahman A, McLeod HL, Scott EP, Lin JS. Genetic basis for a lower prevalence of deficient CYP2D6 oxidative drug metabolism phenotypes in black 
Americans. The Journal of clinical investigation. 1993;91(5):2150-4.

59.Bradford LD. CYP2D6 allele frequency in European Caucasians, Asians, Africans and their descendants. Pharmacogenomics. 2002;3(2):229-43.

60.US FDA. US product monograph for Prilosec (omeprazole) delayed-release capsules and Prilosec (omeprazole magnesium) for delayed-release oral suspension. Last accessed 26 June 2020. at:

https://www.accessdata.fda.gov/drugsatfda_d ocs/label/2012/019810s096lbl.pdf.

61.Adkin DA, Davis SS, Sparrow RA, Huckle PD, Wilding IR. The effect of mannitol on the oral bioavailability of cimetidine. Journal of pharmaceutical sciences. 1995;84(12):1405-9.

62.Health Canada, Cordarone ${ }^{\circledR}$ (Amiodarone $\mathrm{HCl})$ Tablet Label [Online]. Last Accessed 27 March 2019, at: https://pdf.hres.ca/dpd pm/00033875.PDF.

63.Pauli-Magnus C, Rekersbrink S, Klotz U, Fromm MF. Interaction of omeprazole, lansoprazole and pantoprazole with Pglycoprotein. Naunyn-Schmiedeberg's archives of pharmacology. 2001;364(6):5517.

64.Garcia-Arieta A, Gordon J. Bioequivalence requirements in the European Union: critical discussion. Aaps j. 2012;14(4):738-48.

65. Vaithianathan S, Haidar SH, Zhang X, Jiang W, Avon C, Dowling TC, et al. Reply to "On the Effect of Common Excipients on the Oral Absorption of Class 3 Drugs". Journal of pharmaceutical sciences. 2016;105(4):1355-7.
66.US-FDA, CDER: Guidance for Industry, Bioavailability and Bioequivalence Studies for Orally Administered Drug Products General Considerations. July 2002. Last accessed 13 March 2019 at https://www.fda.gov/downloads/Drugs/Guida nces/ucm 154838 .

67.van Os S, Relleke M, Piniella PM. Lack of bioequivalence between generic risperidone oral solution and originator risperidone tablets. International journal of clinical pharmacology and therapeutics. 2007;45(5):293-9.

68. Trifiro G, Parrino F, Sultana J, Giorgianni F, Ferrajolo C, Bianchini E, et al. Drug interactions with levothyroxine therapy in patients with hypothyroidism: observational study in general practice. Clinical drug investigation. 2015;35(3):187-95.

69. Meredith P. Bioequivalence and other unresolved issues in generic drug substitution. Clinical therapeutics. 2003;25(11):2875-90.

70. Volans GN. Migraine and drug absorption. Clinical pharmacokinetics. 1978;3(4):313-8.

71.Centanni M, Gargano L, Canettieri G, Viceconti N, Franchi A, Delle Fave G, et al. Thyroxine in goiter, Helicobacter pylori infection, and chronic gastritis. The New England journal of medicine. 2006;354(17):1787-95.

72.Health Canada, ${ }^{\text {PrIsoptin } ® ~ S R ~ V e r a p a m i l ~ H C l ~}$ Tablets Drug Product Monograph, 2016. [Online]. Last accessed 30 March 2019, at: http://www.mylan.ca/-

Imedia/mylanca/documents/english/product $\%$ 20pdf/pdfs\%20dec\%202015/isoptin-pm2016.01.08.pdf 